\title{
Conformal maps from a 2-torus to the 4-sphere
}

\author{
By Christoph Bohle at Berlin, Katrin Leschke at Leicester, \\ Franz Pedit at Tübingen and Amherst, and Ulrich Pinkall at Berlin
}

\begin{abstract}
We study the space of conformal immersions of a 2-torus into the 4-sphere. The moduli space of generalized Darboux transforms of such an immersed torus has the structure of a Riemann surface, the spectral curve. This Riemann surface arises as the zero locus of the determinant of a holomorphic family of Dirac type operators parameterized over the complexified dual torus. The kernel line bundle of this family over the spectral curve describes the generalized Darboux transforms of the conformally immersed torus. If the spectral curve has finite genus, the kernel bundle can be extended to the compactification of the spectral curve and we obtain a linear 2-torus worth of algebraic curves in projective 3-space. The original conformal immersion of the 2-torus is recovered as the orbit under this family of the point at infinity on the spectral curve projected to the 4-sphere via the twistor fibration.
\end{abstract}

\section{Introduction}

Much of differential geometry, as we know today, has its origins in the theory of surfaces. Initially, this theory had been local, example oriented, and focused on special surface classes in $\mathbb{R}^{3}$. This changed in the middle of the twentieth century when global questions about surfaces in $\mathbb{R}^{3}$ were considered, e.g., do there exists complete or compact surfaces in $\mathbb{R}^{3}$ with special properties such as constant mean or Gaussian curvatures? The integrability conditions for a surface in $\mathbb{R}^{3}$ are a system of non-linear partial differential equations and those questions led to significant advances in the global analysis of geometrically defined partial differential equations. Aside from minimal surfaces, whose analysis is essentially governed by the Cauchy-Riemann equation, significant progress has been made in the description of (non-zero) constant mean curvature surfaces and Willmore surfaces. These are solutions to the elliptic variational problems for area under constrained volume, and for the bending, or Willmore, energy $\int H^{2}$ given by averaging the mean curvature square over the surface.

For constant mean curvature surfaces the combination of non-linear elliptic analysis and methods from integrable system theory goes some way towards describing the moduli spaces of such surfaces of a given topology. A particularly fortuitous situation occurs for constant mean curvature tori: those are the periodic orbits of an algebraically completely 
integrable system whose phase space is the universal Jacobian over an appropriate moduli of compact Riemann surfaces [21], [15], and [2].

The analysis of Willmore surfaces turns out to be more complicated since the integrability condition is a system of non-linear fourth order partial differential equations. Results are less complete and details not all worked out when compared to the constant mean curvature case. Nevertheless, Willmore tori which are not Möbius congruent to Euclidean minimal surfaces are still given by an algebraically completely integrable system (cf. [22], [3]).

That surfaces of genus one should play such a prominent role can be seen in a number of ways. A constant mean curvature surface, and also a Willmore surface, can be described by a circle family of flat $\mathbf{S U}(2)$ respectively $\mathbf{S p}(2)$-connections. Complexifying, one obtains a meromorphic $\mathbb{P}^{1}$-family of $\mathbf{S l}(2, \mathbb{C})$ respectively $\mathbf{S p}(2, \mathbb{C})$-connections with simple poles at 0 and $\infty$ in $\mathbb{P}^{1}$. On a surface of genus one the holonomy representation of this family of flat connections, with respect to a chosen base point on the torus, is abelian. The characteristic polynomial of any of the generators of the holonomy thus gives rise to the same Riemann surface, the spectral curve, which is a double, respectively quadruple, branched cover over $\mathbb{C}_{*}=\mathbb{P}^{1} \backslash\{0, \infty\}$. The ellipticity of the two variational problems allows one to compactify the spectral curve (cf. [15], [3]) to a double, respectively quadruple, branched cover of the Riemann sphere $\mathbb{P}^{1}$. The spectral curve has a real structure induced by the complexification of the circle family to a $\mathbb{C}_{*}$-family of flat connections.

The eigenlines of the $\mathbb{C}_{*}$-family of holonomies extend to a holomorphic line bundle, the eigenline bundle, over the compactified spectral curve. Changing the base point on the torus, with respect to which the holonomy representations were computed, the $\mathbb{C}_{*}$-family of holonomies gets conjugated and thus the spectral curve is left unchanged. What does change is the holomorphic isomorphism type of the eigenline bundle due to contributions from the simple poles at 0 and $\infty$ of the $\mathbb{C}_{*}$-family of flat connections. In fact, the eigenline bundles for the various base points on the torus describe a real 2-dimensional subtorus in the Jacobian of the spectral curve. It turns out that this subtorus is tangent to the real part of the Abel image of the spectral curve inside the Jacobian at the origin.

What we have just described is known in the mathematical physics literature as finite gap integration. The integrability conditions for constant mean curvature and Willmore tori in $\mathbb{R}^{3}$, the zero-curvature equations of the corresponding families of flat connections, are the elliptic affine Toda field equations for the groups $\mathbf{S U}(2)$ - the elliptic sinh-Gordon equation - and $\mathbf{S p}(2)$ respectively (cf. [12], [5]). Such equations, often referred to as soliton equations, are studied in mathematical physics and are known to have an infinite hierarchy of commuting flows. The crucial observation here is that at a doubly periodic solution those flows span a finite dimensional space (cf. [21], [7]), which turns out to be the Jacobian of our previous description. The osculating flag to the Abel image of the spectral curve inside the Jacobian at the origin describes the hierarchy of flows modulo lower order flows. The importance of finite gap solutions, which include doubly periodic solutions, comes from their explicit nature: these solutions can be written down in terms of theta functions on the corresponding spectral curve. As a consequence, one obtains theta function parameterizations of constant mean curvature tori [2] and, in principle, also of Willmore tori.

Despite this rather complete picture of the moduli spaces of constant mean curvature and Willmore tori, a number of basic questions remain unanswered. For instance, what are 
the minimal values of the two variational problems and on which tori are they achieved? A well-known conjecture, first formulated by Willmore, states that the minimal value of the bending energy $\int H^{2}$ over immersed tori is $2 \pi^{2}$ and is attained on the Clifford torus in the 3 -sphere. Note that the bending energy is invariant under the larger group of Möbius transformations of $\mathbb{R}^{3}$. Restricting to constant mean curvature surfaces, which themselves are not Möbius invariant, the bending energy becomes the area functional and one deals with area estimates of constant mean curvature tori. In this context one should also mention the conjecture, due to Lawson, that the Clifford torus in $S^{3}$ is the only embedded minimal torus. By using techniques from integrable system theory together with quaternionic holomorphic geometry, some progress has been made towards a resolution of these conjectures (cf. [22], [11], [16]).

Instead of focusing on the "classical" solutions of the above action functionals on surfaces, this paper shifts attention to the study of the moduli space of all conformal immersions of a Riemann surface. This idea is reminiscent to the path integral quantization in physics where one averages over the space of all contending fields rather than just the classical configurations given by the critical points of the action. Describing this more fundamental space of all conformal immersions of a Riemann surface also sheds new light on the constructions of constant mean curvature and Willmore tori.

Even though one is traditionally interested in surfaces in $\mathbb{R}^{3}$, our constructions necessitate to also consider surfaces in $\mathbb{R}^{4}$. The conformality condition for an immersed surface is invariant under Möbius transformations and thus we are concerned with the space $\mathscr{M}$ of (branched) conformal immersions $f: M \rightarrow S^{4}$ of a Riemann surface $M$ into the 4-sphere $S^{4}$ up to Möbius equivalence. A special situation arises for oriented branched conformal immersions of a compact Riemann surface $M$ taking values in $S^{2} \subset S^{4}$ : these are the meromorphic functions on $M$ which can all be written down in terms of theta functions on $M$. Projections of holomorphic curves in $\mathbb{P}^{3}$ via the twistor map to $S^{4}$ result in branched conformal immersions $f: M \rightarrow S^{4}$ into the 4-sphere which, in fact, are Willmore surfaces. These make up a portion of the moduli space $\mathscr{M}$ described by the meromorphic functions on $M$. Generally though, a conformal immersion $f: M \rightarrow S^{4}$ will not be of this simple type and it is exactly those which are of interest in the present paper.

To provide some intuition how we view the moduli space of conformal immersions, let us assume that $\mathscr{M}$ is a phase space of an integrable system similar to the ones described above. If this were the case, we would have to see abelian groups - the energy shells of the integrable system - acting on the phase space. The least we should see though are Darboux transformations: these are transformations on $\mathscr{M}$ which obey Bianchi permutability and thus span an abelian group. In the aforementioned example of a constant mean curvature torus $f: T^{2} \rightarrow \mathbb{R}^{3}$, this abelian group is the Jacobian of its spectral curve. The Darboux transforms are the secants to the Abel image at the origin which span the Jacobian. In this way we can think of the spectral curve, or rather its Abel image, as the space of all Darboux transformations. This suggests that finding a notion of Darboux transformations for a general conformal immersion $f: M \rightarrow S^{4}$ will allow us to construct an analogue of the spectral curve.

Historically, there is the classical Darboux transformation of isothermic surfaces (cf. [8], [14]): two conformally immersed surfaces $f, f^{\#}: M \rightarrow S^{4}$ are classical Darboux transforms of each other if there exists a 2-sphere congruence $S$, that is, $S(p)$ is an oriented 
round 2-sphere in $S^{4}$, touching $f$ and $f^{\#}$ at $p \in M$. Darboux showed that if two surfaces are related by a classical Darboux transform, then both surfaces have to be isothermic, meaning that the principal curvature lines of the surface are conformal coordinates on $M$.

In order to study general conformal immersions $f: M \rightarrow S^{4}$, we have to relax the touching conditions which characterize Darboux transforms: instead of demanding that the 2-sphere congruence $S$ touches both surfaces $f$ and $f^{\#}$, we only demand that $S$ touches $f$ and left-touches $f^{\#}$ at the corresponding points. Two oriented planes through the origin in $\mathbb{R}^{4}$ are left-touching if their oriented intersection great circles in $S^{3} \subset \mathbb{R}^{4}$ correspond under right multiplication by $S^{3}$. Note that even if the original surface $f$ is contained in 3-space, the Darboux transform $f^{\#}$ has to lie in 4-space to avoid both surfaces to be isothermic: left-touching planes in $\mathbb{R}^{3}$ automatically coincide. This explains the necessity to study conformal immersions into 4-space even if one is mainly concerned about surfaces in 3-space. The Darboux transformation so defined is invariant under Möbius transformations and satisfies Bianchi permutability: if $f^{\#}$ and $f^{b}$ are Darboux transforms of $f: M \rightarrow S^{4}$, then there is a conformal map $\hat{f}: M \rightarrow S^{4}$ which is a Darboux transform of $f^{\#}$ and $f^{b}$.

Following our intuitive reasoning, we view the space of all Darboux transforms $f^{\#}: M \rightarrow S^{4}$ of a conformal immersion $f: M \rightarrow S^{4}$ as an analog of the spectral curve. Our aim is to show that under certain circumstances this space indeed has the structure of a Riemann surface.

We model the Möbius geometry of the 4-sphere by the projective geometry of the quaternionic projective line $\mathbb{M} \mathbb{P}^{1}$. A map $f: M \rightarrow \mathbb{P} \mathbb{P}^{1}$ is given by the quaternionic line subbundle $L \subset V$ of the trivial $\mathbb{H}^{2}$-bundle $V$ with fiber $L_{p}=f(p) \subset \mathbb{H}^{2}$ for $p \in M$. Conformality of $f$ is expressed by the existence of a (quaternionic) holomorphic structure, that is, a first order elliptic operator

$$
D: \Gamma(V / L) \rightarrow \Gamma(\bar{K} V / L)
$$

on the line bundle $V / L$. The kernel $H^{0}(V / L)$ of $D$ defines the space of (quaternionic) holomorphic sections of $V / L$ which contains the 2-dimensional linear system $H \subset H^{0}(V / L)$ obtained by projecting constant sections in $V$ modulo $L$. This linear system gives rise to the conformal immersion $f$ via the Kodaira correspondence (A.8).

In order to obtain an analytic description of Darboux transformations, we show that a Darboux transform $f^{\#}$ of $f$ corresponds to a non-trivial holomorphic section $\psi$ with monodromy of $V / L$, that is, a section $\psi$ on the universal cover of $M$ satisfying

$$
D \psi=0 \quad \text { and } \quad \gamma^{*} \psi=\psi h_{\gamma}
$$

for some representation $h: \pi_{1}(M) \rightarrow \mathbb{H}_{*}$ of the fundamental group of $M$. Therefore, to describe the space of Darboux transforms of $f$, we have to characterize the subspace of possible monodromies $h \in \operatorname{Hom}\left(\pi_{1}(M), \mathbb{H}_{*}\right)$ up to conjugation by $\mathbb{H}_{*}$ of the holomorphic structure $D$ on $V / L$.

At this juncture we specialize to the situation when $M=T^{2}$ is a 2-torus and $f: T^{2} \rightarrow S^{4}$ is a conformal immersion whose normal bundle has degree zero. Then the fundamental group $\pi_{1}\left(T^{2}\right)$ is a rank 2 lattice $\Gamma \subset \mathbb{R}^{2}$ and every representation $h \in \operatorname{Hom}\left(\Gamma, \mathbb{M}_{*}\right)$ 
can be conjugated to a complex representation unique up to complex conjugation. The subspace

$$
\operatorname{Spec}(V / L, D) \subset \operatorname{Hom}\left(\Gamma, \mathbb{C}_{*}\right)
$$

of possible complex monodromies of $D$ on $V / L$ over the torus $T^{2}$ is called the spectrum of $D$. Here we make contact to Floquet theory of the periodic operator $D$ outlined by Taimanov [23], Grinevich and Schmidt [13], [22] in their approach to the spectral curve. Conjugating the operator $D$ by the function $e^{\int \omega}$ on $\mathbb{R}^{2}$, where $\omega \in \operatorname{Harm}\left(T^{2}, \mathbb{C}\right)$ is a harmonic 1-form, we obtain a holomorphic family

$$
D_{\omega}: \Gamma(V / L) \rightarrow \Gamma(\bar{K} V / L)
$$

of first order elliptic operators parameterized over $\operatorname{Harm}\left(T^{2}, \mathbb{C}\right) \cong \mathbb{C}^{2}$. The kernel of $D_{\omega}$ describes precisely the holomorphic sections of $D$ with monodromy $h=e^{\int \omega}$. Since the normal bundle degree of the immersion $f: T^{2} \rightarrow S^{4}$ is zero the family $D_{\omega}$ has index zero. This implies [4] that the spectrum $\operatorname{Spec}(V / L, D) \subset \operatorname{Hom}\left(\Gamma, \mathbb{C}_{*}\right)$ is a 1-dimensional analytic subvariety given by the zero locus of the determinant $\operatorname{det} D_{\omega}$ of the holomorphic family $D_{\omega}$. Moreover, the kernel of $D_{\omega}$ along the spectrum is generically 1-dimensional. The normalization $h: \Sigma \rightarrow \operatorname{Spec}(V / L, D)$ of the spectrum to a Riemann surface is the spectral curve $\Sigma$ of the immersed torus $f: T^{2} \rightarrow S^{4}$. It carries a fixed point free real structure $\rho$ induced by complex conjugation on the spectrum $\operatorname{Spec}(V / L, D)$. The kernels ker $D_{\omega}$ give rise to a complex holomorphic line bundle $\mathscr{L}$ over $\Sigma$ whose fiber $\mathscr{L}_{x}$ over $x \in \Sigma$ is spanned by a holomorphic section $\psi$ of $V / L$ with monodromy $h(x)$. Such a section gives rise to a Darboux transform $f^{\#}$ of $f$. Therefore $\Sigma / \rho$ parameterizes the space of generic Darboux transforms of the conformal immersion $f: T^{2} \rightarrow S^{4}$.

Analyzing the behavior of the holomorphic family $D_{\omega}$ for large monodromies shows (cf. [4]) that the spectrum $\operatorname{Spec}(V / L, D)$ is asymptotic to the vacuum spectrum $\operatorname{Spec}(V / L, \bar{\partial})$ belonging to the complex linear part $\bar{\partial}$ of $D$ on $V / L$. The vacuum spectrum $\operatorname{Spec}(V / L, \bar{\partial}) \subset \operatorname{Hom}\left(\Gamma, \mathbb{C}_{*}\right)$ is a singular curve isomorphic to $\mathbb{C} \cup \mathbb{C}$ with the $\mathbb{Z}^{2}$-lattice of real representations as double points. Therefore, the normalization of the vacuum spectrum can be compactified to two copies of $\mathbb{P}^{1}$ by adding two points at infinity and the real structure $\rho$ exchanges the two components.

To summarize, we have associated to a conformal immersion $f: T^{2} \rightarrow S^{4}$ from a 2-torus to the 4-sphere with zero normal bundle degree a Riemann surface $\Sigma$ with either one or two ends, the spectral curve of $f$. Depending on whether $\Sigma$ has one or two ends, the genus of $\Sigma$ is infinite or finite. The spectral curve has a fixed point free real structure $\rho$ induced by complex conjugation on the spectrum $\operatorname{Spec}(V / L, D)$. The kernels of $D_{\omega}$ define a complex holomorphic line bundle $\mathscr{L}$ over $\Sigma$ of quaternionic type, that is, $\rho^{*} \mathscr{L} \cong \overline{\mathscr{L}}$. The fibers of $\mathscr{L}$ over $x \in \Sigma$ describe Darboux transformations $f^{x}$ of $f$, whereby fibers over $x$ and $\rho(x)$ give rise to the same Darboux transform. The resulting map

$$
F: T^{2} \times \Sigma \rightarrow S^{4}, \quad F(p, x)=f^{x}(p)
$$

is conformal in the first factor and lifts, via the twistor projection $\mathbb{P}^{3} \rightarrow S^{4}$, to a map

$$
\hat{F}: T^{2} \times \Sigma \rightarrow \mathbb{P}^{3}
$$

which is holomorphic in the second factor. 
As an example, we consider homogeneous tori $f: T^{2} \rightarrow S^{4}$ given by the products of two circles of varying radii. The holomorphic structure $D=\bar{\partial}+Q$ on $V / L$ has constant $Q$ in an appropriate trivialization. The spectrum $\operatorname{Spec}(V / L, D)$ is a perturbation of the vacuum spectrum $\operatorname{Spec}(V / L, \bar{\partial})$ by $Q$ which has the effect that one of the double points of $\operatorname{Spec}(V / L, \bar{\partial})$ resolves into a handle. Consequently, $\operatorname{Spec}(V / L, D)$ is connected and its normalization $\Sigma$ compactifies to the Riemann sphere $\mathbb{P}^{1}$ by adding two points $o$ and $\infty=\rho(o)$ at infinity. The map $\hat{F}$ extends holomorphically into the points at infinity and is given by a certain $T^{2}$-family of rational cubics. Therefore, the map $F$, obtained from $\hat{F}$ by twistor projection, yields a $T^{2}$-family of Möbius congruent Veronese embeddings of $\mathbb{R} \mathbb{P}^{2}=\mathbb{P}^{1} / \rho$ in $S^{4}$. The original homogeneous torus $f$ is recovered by evaluating this family at infinity, $f=F(-, o)=F(-, \infty)$.

In contrast to this example, the general conformal immersion $f: T^{2} \rightarrow S^{4}$ of a 2-torus of zero normal bundle degree will not have a spectral curve $\Sigma$ which can be compactified: the holomorphic structure $D=\bar{\partial}+Q$ on $V / L$ is a perturbation of the vacuum $\bar{\partial}$ by some "potential" $Q \in \Gamma(\bar{K} \operatorname{End}(V / L))$ which, based on physical intuition, has the effect that the $\mathbb{Z}^{2}$-lattice of double points of the vacuum $\operatorname{spectrum} \operatorname{Spec}(V / L, \bar{\partial})$ resolves into a $\mathbb{Z}^{2}$-lattice of handles for $\Sigma$ accumulating at infinity. Even though the theory of such Riemann surfaces of infinite genus [10] resembles to some degree the theory of compact Riemann surfaces, it is not algebro-geometric in nature.

This leads us to consider conformal tori $f: T^{2} \rightarrow S^{4}$ of zero normal bundle degree whose spectral curves have finite genus and therefore two ends. In this case only finitely many double points of the vacuum spectrum $\operatorname{Spec}(V / L, \bar{\partial})$ become handles for $\operatorname{Spec}(V / L, D)$. In the mathematical physics literature the corresponding potentials $Q \in \Gamma(\bar{K} \operatorname{End}(V / L))$ with $D=\bar{\partial}+Q$ are called finite gap potentials. The normalization $\Sigma$ of $\operatorname{Spec}(V / L, D)$ can therefore be compactified by the addition of two points $o$ and $\infty=\rho(o)$ at infinity as in the example of homogeneous tori above. The genus of the compactified spectral curve $\bar{\Sigma}$ is called the spectral genus of the conformal torus $f: T^{2} \rightarrow S^{4}$. Important examples of conformal tori of finite spectral genus include, but are not confined to, constant mean curvature [21], [15] and (constrained) Willmore tori [22], [3]. In fact, there is reasonable evidence from analogous situations in the literature [17], [19] that conformal tori of finite spectral genus are dense in the space of all conformal tori of zero normal bundle degree in the 4 -sphere. When $\Sigma$ has finite genus, we show that the map $\hat{F}: T^{2} \times \Sigma \rightarrow \mathbb{P}^{3}$ extends holomorphically in the second component to $T^{2} \times \bar{\Sigma}$. The resulting $T^{2}$-family of algebraic curves in $\mathbb{P}^{3}$, respectively their twistor projections to the 4-sphere, recovers the initial conformal torus $f: T^{2} \rightarrow S^{4}$ of finite spectral genus by evaluation $f=F(-, o)=F(-, \infty)$ at infinity.

At this stage, we have come some way in describing conformal immersions of 2-tori into the 4-sphere with zero normal bundle degree and finite spectral genus in terms of algebro-geometric data: a compact finite genus curve $\bar{\Sigma}$ with fixed point free real structure and a $T^{2}$-family of algebraic curves into $\mathbb{P}^{3}$ compatible with the real structure. As it turns out [4], this $T^{2}$-family is given by linear motion on the real part of the Jacobian of $\bar{\Sigma}$ tangent to the real part of its Abel image at the origin. In fact, this flow is the first in a hierarchy of commuting flows preserving the Willmore energy coming from the osculating flag to the Abel image at the origin. It is precisely this observation which makes the space of conformal maps $f: T^{2} \rightarrow S^{4}$ the phase space of a completely integrable system, containing constant mean curvature and Willmore tori as invariant subspaces. This system is a 
geometric manifestation of the Davey-Stewartson [9] hierarchy known to mathematical physicists and what we have described is a geometric version of its finite gap integration theory.

\section{The Darboux transformation}

In this section we introduce the Darboux transformation for conformal immersions $f: M \rightarrow S^{4}$ of a Riemann surface $M$ into the 4-sphere. Similar to Darboux's classical transformation of isothermic surfaces, our transformation of conformal immersions is also given by a non-linear, Möbius geometric touching condition with respect to a 2 -sphere congruence. Whereas the classical Darboux transformation can be computed by solving a system of linear ordinary differential equations, the general Darboux transformation of conformal maps is described analytically by a quaternionic holomorphicity condition, a linear first order elliptic partial differential equation.

2.1. Conformal maps into $S^{4}$. We model the Möbius geometry of $S^{4}$ by the projective geometry of the (quaternionic) projective line $\mathbb{M P} \mathbb{P}^{1}$. Thus, a map $f: M \rightarrow S^{4}$ is given by the line subbundle $L \subset V$ of the trivial $\mathbb{H}^{2}$-bundle $V$ over $M$, where the fiber $L_{p}$ over $p \in M$ is the projective point $L_{p}=f(p)$. In other words, $L=f^{*} \mathscr{T}$ is the pullback under $f$ of the tautological line bundle $\mathscr{T}$ over $\mathbb{M P} \mathbb{P}^{1}$. Since the tangent bundle of $\mathbb{H} \mathbb{P}^{1}$ is $\operatorname{Hom}\left(\mathscr{T}, \underline{\mathbb{Q}}^{2} / \mathscr{T}\right)$, the derivative of $f$ corresponds to the 1 -form

$$
\delta=\left.\pi d\right|_{L} \in \Omega^{1}(\operatorname{Hom}(L, V / L)) .
$$

Here $d$ denotes the trivial connection on $V$ and $\pi: V \rightarrow V / L$ is the canonical projection.

To describe the conformality of $f$ note (cf. [6], [20]) that over immersed points $p \in M$ the real 2-plane $\delta\left(T_{p} M\right) \subset \operatorname{Hom}\left(L_{p}, \mathbb{M}^{2} / L_{p}\right)$ is given by

$$
J_{p} X=X \tilde{J}_{p}, \quad X \in \operatorname{Hom}\left(L_{p}, \mathbb{T}^{2} / L_{p}\right)
$$

for uniquely existing complex structures $J_{p}$ on $\mathbb{H}^{2} / L_{p}$ and $\tilde{J}_{p}$ on $L_{p}$ which are compatible with the orientation on $\delta(T M)$ induced from the Riemann surface $M$. Therefore, if $*$ denotes the complex structure on $T M^{*}$, the conformality equation for $f$ over immersed points reads

$$
* \delta=J \delta=\delta \tilde{J} .
$$

In what follows, we consider conformal maps $f: M \rightarrow S^{4}$ for which at least one of the complex structures $J$ or $\tilde{J}$ extends smoothly across the branch points. This class of conformal maps, which includes conformal immersions, can be described in terms of quaternionic holomorphic geometry [11]. Notice that the point-point duality of $\mathbb{M} \mathbb{P}^{1}$ exchanges $L \subset V$ with $L^{\perp} \subset V^{*}$. Therefore, a conformal map $f$ with $* \delta=J \delta$ becomes, via this duality, the conformal map $f^{\perp}$ with $* \delta^{\perp}=\delta^{\perp} J^{*}$, where we identify $V / L=\left(L^{\perp}\right)^{*}$ and $\delta^{\perp}=-\delta^{*}$.

An important invariant of a conformal map $f$ with $* \delta=J \delta$ is its associated quaternionic holomorphic structure (A.3) on the line bundle $V / L$. This structure is given by the first order linear elliptic operator

$$
D: \Gamma(V / L) \rightarrow \Gamma(\bar{K} V / L) \quad \text { defined by } D \pi=(\pi d)^{\prime \prime},
$$


where $K$ denotes the canonical bundle of the Riemann surface $M$. The operator $D$ is well defined since $\left.\pi d\right|_{L}=\delta \in \Gamma(K \operatorname{Hom}(L, V / L))$ and thus $\left(\left.\pi d\right|_{L}\right)^{\prime \prime}=0$. If $f$ is non-constant, the canonical projection $\pi$ realizes $\mathbb{T}^{2}$ as a 2-dimensional linear system $H \subset H^{0}(V / L)$ whose Kodaira embedding (A.8) is $L^{\perp} \subset V^{*}$.

The Willmore energy of the conformal map $f$ is given by the Willmore energy (A.6) of the holomorphic line bundle $V / L$, that is,

$$
\mathscr{W}(f)=\mathscr{W}(V / L, D)=2 \int_{M}\langle Q \wedge * Q\rangle
$$

where $Q \in \Gamma\left(\bar{K}\right.$ End $\left._{-}(V / L)\right)$ is the $J$-anticommuting part of $D$.

If $f: M \rightarrow S^{4}$ is an immersion, also $L \subset V$ has a complex structure $\tilde{J}$ and $* \delta=J \delta=\delta \tilde{J}$ by (2.1). In particular, $\operatorname{Hom}_{+}(L, V / L)$ is the tangent bundle and $N_{f}=\operatorname{Hom}_{-}(L, V / L)$ the normal bundle of $f$, where $\operatorname{Hom}_{ \pm}(L, V / L)$ denote the complex linear, respectively complex antilinear, homomorphisms. Since $\delta \in \Gamma\left(K \operatorname{Hom}_{+}(L, V / L)\right)$ is a complex bundle isomorphism, the normal bundle degree of the conformal immersion $f: M \rightarrow S^{4}$, in case $M$ is compact, calculates to

$$
\operatorname{deg} N_{f}=2 \operatorname{deg} V / L+\operatorname{deg} K,
$$

where the degree of a quaternionic bundle is defined in (A.2). As shown in [6], Proposition 11, the Möbius invariant 2-form $2\langle Q \wedge * Q\rangle$, the integrand for the Willmore energy (2.3), coincides over immersed points of $f$ with

$$
2\langle Q \wedge * Q\rangle=\left(|\mathscr{H}|^{2}-\mathscr{K}-\mathscr{K}^{\perp}\right) d \mathscr{A} .
$$

Here we have chosen a point at infinity $L_{0} \in S^{4}$ and $\mathscr{H}$ is the mean curvature vector, $\mathscr{K}$ the Gaussian curvature, $\mathscr{K}^{\perp}$ the normal bundle curvature, and $d \mathscr{A}$ the induced area of $f$ as a map into $\mathbb{R}^{4}=S^{4} \backslash\left\{L_{0}\right\}$. Since $V=L \oplus L_{0}$ and $V / L \cong L_{0}$, the trivial connection $d$ on $V$ restricts to a flat connection $\nabla$ on $V / L$ for which $\nabla^{\prime \prime}=D=\bar{\partial}+Q$. Let $\nabla=\hat{\nabla}+A+Q$ be the decomposition of $\nabla$ into the $J$-commuting part $\hat{\nabla}$ and the $J$-anticommuting part $A+Q \in \Omega^{1}(\operatorname{End}(V / L))$, which we have further decomposed into type. If we denote by $\hat{R}$ the curvature of the complex connection $\hat{\nabla}$, then flatness of $\nabla$ implies

$$
J \hat{R}=\langle A \wedge * A\rangle-\langle Q \wedge * Q\rangle .
$$

From [6], Proposition 8, we see that

$$
2\langle A \wedge * A\rangle=|\mathscr{H}|^{2} d \mathscr{A}
$$

over immersed points of $f$. Thus, by $(2.5)$ the 2 -form $\left(\mathscr{K}+\mathscr{K}^{\perp}\right) d \mathscr{A}$ is the Chern form of the bundle $V / L$, the classical Willmore integrand $|\mathscr{H}|^{2} d \mathscr{A}$ extends smoothly into the branch points of $f$, and

$$
\int_{M}|\mathscr{H}|^{2} d \mathscr{A}=\mathscr{W}(f)+4 \pi \operatorname{deg}(V / L) .
$$


Lemma 2.1. Let $f: M \rightarrow S^{4}$ be a non-constant conformal map with $* \delta=J \delta$ of $a$ compact Riemann surface $M$ into $S^{4}$. If the classical Willmore energy of $f$ as a map into $\mathbb{R}^{4}$ satisfies

$$
\int_{M}|\mathscr{H}|^{2} d \mathscr{A}<8 \pi
$$

then $f$ is a conformal embedding with trivial normal bundle. Moreover, $\operatorname{dim} H^{0}(V / L)=2$ which means that all holomorphic sections $\psi \in H^{0}(V / L)$ are of the form $\psi=\pi(v)$ with $v \in \mathbb{M}^{2}$, where $\pi: V \rightarrow V / L$ denotes the canonical projection.

Proof. The proof is a repeated application of the Plücker formula (A.10) for 1-dimensional linear systems $H \subset H^{0}(V / L)$ which, under the assumption of the lemma together with (2.6), satisfy

$$
\text { ord } H<2 \text {. }
$$

If $\operatorname{dim} H^{0}(V / L)>2$, then the linear map $H^{0}(V / L) \rightarrow \mathbb{Q}^{2} / L_{p} \oplus \mathbb{U}^{2} / L_{q}$ given by evaluation of sections at $p \neq q \in M$ has at least a 1-dimensional kernel $H \subset H^{0}(V / L)$. This means that $H$ contains a holomorphic section vanishing at $p$ and $q$ and thus ord $H \geqq 2$.

Next we show that $f$ is injective. If this is not the case, there are distinct points $p \neq q$ on $M$ with $L_{p}=L_{q} \subset \mathbb{H}^{2}$. Choosing any non-zero $v \in L_{p}$, we see that the corresponding non-trivial holomorphic section $\psi=\pi(v)$ of $V / L$ vanishes at $p$ and $q$ and we again have ord $H \geqq 2$.

To prove that $f$ is an immersion, we have to show that $\delta \in \Gamma(K \operatorname{Hom}(L, V / L))$ has no zeros. If $\delta$ has a zero at $p \in M$, we construct a non-trivial holomorphic section $\psi \in H^{0}(V / L)$ which vanishes to second order at $p$ : let $\psi$ be the holomorphic section of $V / L$ given by $\psi=\pi(v)$, where $v \in L_{p} \subset \mathbb{M}^{2}$ is non-zero. Then $\psi$ has a zero at $p \in M$ and for any $\alpha \in \Gamma\left(L^{\perp}\right)$ we get

$$
d_{p}\langle\alpha, \psi\rangle=d_{p}\langle\alpha, v\rangle=\left\langle d_{p} \alpha, v\right\rangle=\left\langle\pi^{\perp} d_{p} \alpha, v\right\rangle=\left\langle\delta_{p}^{\perp}(\alpha), v\right\rangle=0
$$

where we used that $\delta^{\perp}=-\delta^{*}$ and thus $\delta_{p}^{\perp}=0$. This shows that $\psi \in H^{0}(V / L)$ vanishes to second order at $p \in M$ and therefore ord $H \geqq 2$.

The normal bundle degree of $f$ is the self-intersection number of $f(M)$, which is zero since $f$ is an embedding.

2.2. Darboux transforms. An oriented round 2-sphere in $S^{4}=\mathbb{M} \mathbb{P}^{1}$ is given by a linear map $S: \mathbb{H}^{2} \rightarrow \mathbb{H}^{2}$ which has $S^{2}=-1$ : points on the 2-sphere are the fixed lines of $S$. The resulting line subbundle $L_{S} \subset V$ of the trivial $\mathbb{\boxplus}^{2}$-bundle over the 2-sphere satisfies $S L_{S}=L_{S}$. Thus, we have complex structures on $L_{S}$ and $V / L_{S}$ and the conformality equation (2.1) of the embedded round sphere $S$ is $* \delta_{S}=S \delta_{S}=\delta_{S} S$.

Given a Riemann surface $M$, a sphere congruence assigns to each point $p \in M$ an oriented round 2-sphere $S(p)$ in $S^{4}$. In other words, a sphere congruence is a complex structure $S \in \Gamma(\operatorname{End}(V))$ on the trivial $\mathbb{\boxplus}^{2}$-bundle $V$ over $M$. 
Now let $f: M \rightarrow S^{4}$ be a conformal map with induced line bundle $L=f^{*} \mathscr{T} \subset V$. A sphere congruence $S \in \Gamma(\operatorname{End}(V))$ envelopes $f$ if for all points $p \in M$ the spheres $S(p)$ pass through $f(p)$, and the oriented tangent spaces to $f$ coincide with the oriented tangent spaces to the spheres $S(p)$ at $f(p)$ over immersed points $p \in M$ :

$$
S L=L \quad \text { and } \quad * \delta=S \delta=\delta S .
$$

It is a classical result (cf. [8], [14]) that two distinct conformal immersions $f$ and $f^{\#}$ from the same Riemann surface $M$ which are both enveloped by the same sphere congruence $S$ have to be isothermic surfaces. To overcome this restriction, we need to relax the enveloping condition: two oriented planes through the origin in $\mathbb{R}^{4}$ are left-touching, respectively right-touching, if their associated oriented great circles on $S^{3}$ correspond via right, respectively left, translation in the group $S^{3}$. Hence, we say that a sphere congruence $S$ leftenvelopes, respectively right-envelopes, $f$ if for all points $p \in M$ the spheres $S(p)$ pass through $f(p)$, and the oriented tangent spaces to $f$ are left-touching, respectively righttouching, to the oriented tangent spaces to the spheres $S(p)$ at $f(p)$ over immersed points $p \in M$ :

$$
S L=L \quad \text { and } \quad * \delta=S \delta, \quad \text { respectively } \quad * \delta=\delta S
$$

Definition 2.2. Let $M$ be a Riemann surface. A conformal map $f^{\#}: M \rightarrow S^{4}$ is called a Darboux transform of a conformal immersion $f: M \rightarrow S^{4}$ if $f^{\#}(p)$ is distinct from $f(p)$ at all points $p \in M$, and if there exists a sphere congruence $S$ which envelopes $f$ and left-envelopes $f^{\#}$ :

$$
V=L \oplus L^{\#}, \quad S L=L, \quad * \delta=S \delta=\delta S, \quad \text { and } \quad S L^{\#}=L^{\#}, \quad * \delta^{\#}=S \delta^{\#} .
$$

In particular, if $f$ and $f^{\#}$ are conformal immersions into $S^{3}$ and $f^{\#}$ is a Darboux transform of $f$, then both $f$ and $f^{\#}$ are isothermic. This follows from the fact that in 3 -space a half-enveloping sphere congruence is always enveloping.

There are a number of equivalent characterizations of Darboux transforms $f^{\#}: M \rightarrow S^{4}$ of a conformal immersion $f: M \rightarrow S^{4}$, including a description in terms of flat adapted connections and, more generally, holomorphic sections with monodromy of the bundle $V / L$. It is this last analytic characterization which will play a fundamental role in our construction of the spectral curve.

Let $f, f^{\#}: M \rightarrow S^{4}$ be maps distinct from each other at all points $p \in M$. Then $V=L \oplus L^{\#}$ and $\pi$ identifies $V / L=L^{\#}$ whereas $\pi^{\#}$ identifies $V / L^{\#}=L$. The trivial connection $d$ on $V$ decomposes as

$$
d=\left(\begin{array}{cc}
\nabla^{L} & \delta^{\#} \\
\delta & \nabla^{\#}
\end{array}\right)
$$

and flatness of $d$ implies that

$$
d \delta=0, \quad d \delta^{\#}=0, \quad \text { and } \quad R^{\#}=-\delta \wedge \delta^{\#},
$$

where $R^{\#}$ denotes the curvature of $\nabla^{\#}$. 
Lemma 2.3. Let $f: M \rightarrow S^{4}$ be a conformal immersion with $* \delta=J \delta=\delta \tilde{J}$ and $f^{\#}: M \rightarrow S^{4}$ be a map so that $V=L \oplus L^{\#}$. Then we have the following equivalent characterizations of Darboux transforms:

(i) The map $f^{\#}$ is a Darboux transform of $f$.

(ii) The map $f^{\#}$ is conformal with $* \delta^{\#}=\tilde{J} \delta^{\#}$.

(iii) The connection $\nabla^{\#}$ on $L^{\#}$ induced by the splitting $V=L \oplus L^{\#}$ is flat.

(iv) There is a non-trivial section $\psi^{\#} \in \Gamma\left(\widetilde{L^{\#}}\right)$ with monodromy satisfying $d \psi^{\#} \in \Omega^{1}(\tilde{L})$.

A section $\psi$ with monodromy of a vector bundle $W \rightarrow M$ is a section of the pullback bundle $\tilde{W} \rightarrow \tilde{M}$ of $W$ to the universal cover $\tilde{M} \rightarrow M$ with

$$
\gamma^{*} \psi=\psi h_{\gamma}
$$

where $h: \pi_{1}(M) \rightarrow \mathbb{H}_{*}$ is a representation and $\gamma \in \pi_{1}(M)$ acts as a deck transformation.

Proof. The unique sphere congruence $S$ touching $f$ and containing $f^{\#}$, expressed in the splitting $V=L \oplus L^{\#}$, is

$$
\left(\begin{array}{ll}
\tilde{J} & 0 \\
0 & J
\end{array}\right) .
$$

Therefore, the sphere congruence $S$ left-touches $f^{\#}$ if and only if $* \delta^{\#}=\tilde{J} \delta^{\#}$, which proves the first equivalence by (2.9). The second equivalence is a direct consequence of $R^{\#}=-\delta \wedge \delta^{\#}$ in (2.11) together with a type consideration. The last equivalence follows from (2.10) because flatness of $\nabla^{\#}$ is equivalent to the existence of parallel sections with monodromy of the line bundle $\tilde{L}^{\#}$.

The isomorphism $\pi: L^{\#} \rightarrow V / L$ pushes forward the connection $\nabla^{\#}$ to a connection $\nabla$ on $V / L$ satisfying

$$
\left.\nabla \pi\right|_{\Gamma\left(L^{\#}\right)}=\pi \nabla^{\#}=\left.\pi d\right|_{\Gamma\left(L^{\#}\right)} .
$$

By construction, the connection $\nabla$ is adapted to the complex structure $D$ on $V / L$ defined in (2.2), that is, $\nabla^{\prime \prime}=D$.

For a fixed immersion $f$ the spaces of splittings $V=L \oplus L^{\#}$ and the space of adapted connections on $V / L$ are affine spaces modelled on the vector spaces $\operatorname{Hom}(V / L, L)$ and $\Gamma(K \operatorname{End}(V / L))$, respectively. The map assigning to a splitting the induced adapted connection (2.12) is affine with underlying vector space homomorphism

$$
\Gamma(\operatorname{Hom}(V / L, L)) \rightarrow \Gamma(\Gamma(K \operatorname{End}(V / L))), \quad R \mapsto \delta R
$$

Since $f$ is an immersion and thus $\delta$ an isomorphism, the correspondence assigning to a splitting the induced adapted connection on $V / L$ is an affine isomorphism. Together with 
Lemma 2.3 we get a characterization of Darboux transforms in terms of flat adapted connections on $V / L$.

Corollary 2.4. Let $f: M \rightarrow S^{4}$ be a conformal immersion. Then there is a bijective correspondence between the space of Darboux transforms $f^{\#}: M \rightarrow S^{4}$ of $f$ and the space of flat adapted connections on $V / L$. This correspondence is given by the restriction to the space of Darboux transforms of the affine isomorphism (2.12) assigning to a splitting $V=L \oplus L^{\#}$ the adapted connection $\nabla$ on $V / L$.

In what follows it is necessary to compute Darboux transforms from flat adapted connections by using prolongations of holomorphic sections on $V / L$.

Lemma 2.5. Let $f: M \rightarrow S^{4}$ be a conformal immersion. Then the canonical projection $\pi: V \rightarrow V / L$ induces a bijective correspondence between sections $\hat{\psi} \in \Gamma(V)$ of $V$ satisfying $d \hat{\psi} \in \Omega^{1}(L)$ and holomorphic sections $\psi \in H^{0}(V / L)$.

Proof. Let $\hat{\psi}_{0} \in \Gamma(V)$ be a lift of $\psi \in H^{0}(V / L)$, that is, $\pi \hat{\psi}_{0}=\psi$. Then (2.2) implies $\left(\pi d \hat{\psi}_{0}\right)^{\prime \prime}=D \pi \hat{\psi}_{0}=D \psi=0$ and, since $f$ is an immersion, there is a unique section $\varphi \in \Gamma(L)$ with $\delta \varphi=\left(\pi d \hat{\psi}_{0}\right)$. But then $\hat{\psi}=\hat{\psi}_{0}-\varphi$ is the unique section of $V$ with the required properties.

Definition 2.6. Let $f: M \rightarrow S^{4}$ be a conformal immersion. The prolongation of a holomorphic section $\psi \in H^{0}(V / L)$ is the unique section $\hat{\psi} \in \Gamma(V)$ with $\pi \hat{\psi}=\psi$ satisfying $d \hat{\psi} \in \Omega^{1}(L)$, that is, $\pi d \hat{\psi}=0$.

Given a flat adapted connection $\nabla$ on $V / L$, we want to compute the corresponding Darboux transform of $f$ from Corollary 2.4. We take a parallel section $\psi \in \Gamma(\widetilde{V / L})$ over the universal cover $\tilde{M}$ of $M$. Since $V / L$ is a line bundle this section has monodromy. But $\nabla$ is adapted so that $\psi \in H^{0}(\widetilde{V / L})$ is also a holomorphic section with monodromy, that is,

$$
D \psi=0 \quad \text { and } \quad \gamma^{*} \psi=\psi h_{\gamma}
$$

for a representation $h: \pi_{1}(M) \rightarrow \mathbb{H}_{*}$. Then the prolongation $\hat{\psi} \in \Gamma(\tilde{V})$ is a section with the same monodromy $h$. Moreover, as a parallel section, $\psi$ has no zeros and neither does $\hat{\psi}$. This shows that the line bundle

$$
L^{\#}=\hat{\psi} \boxplus \subset V
$$

is well defined over $M$ and satisfies $V=L \oplus L^{\#}$. As a prolongation, $\hat{\psi}$ has $d \hat{\psi} \in \Omega^{1}(\tilde{L})$, which is one of the equivalent characterizations in Lemma 2.3 for Darboux transforms. This shows that the map $f^{\#}: M \rightarrow S^{4}$ corresponding to the line bundle $L^{\#}$ is the Darboux transform of $f$ belonging to the adapted connection $\nabla$.

The parallel sections of flat adapted connections on $V / L$ are precisely the holomorphic sections with monodromy of $V / L$ that are nowhere vanishing. If a holomorphic section $\psi \in \Gamma(\widetilde{V / L})$ has zeroes, these are isolated [11] and, away from the finite set of zeros, there is a unique flat adapted connection on $V / L$ which makes $\psi$ parallel. Hence, a holomorphic section $\psi$ of $V / L$ with monodromy gives rise to a Darboux transform $f^{\#}$ of $f$

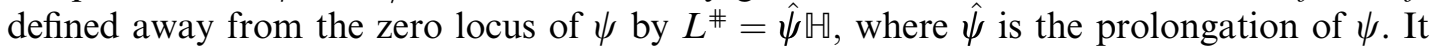


follows from [11], Lemma 3.9, that $f^{\#}$ extends continuously across the zeros of $\psi$ where it agrees with $f$. We call such $f^{\#}$ singular Darboux transforms of $f$.

Lemma 2.7. Let $f: M \rightarrow S^{4}$ be a conformal immersion. Then there is a bijective correspondence between the space of (singular) Darboux transforms $f^{\#}: M \rightarrow S^{4}$ of $f$ and the space of non-trivial holomorphic sections with monodromy up to scale of $V / L$. Under this correspondence, non-singular Darboux transforms get mapped up to scale to nowhere vanishing holomorphic sections with monodromy of $V / L$.

In contrast to Definition 2.2, Lemma 2.3, and Corollary 2.4, which characterize Darboux transforms by non-linear Möbius geometric or zero curvature conditions, Lemma 2.7 characterizes Darboux transforms in terms of solutions to a linear elliptic equation. Therefore, locally $V / L$ has an infinite dimensional space of holomorphic sections without zeros and we get an infinite dimensional space of local Darboux transforms of $f$. The situation is rather different when considering global Darboux transforms $f^{\#}: M \rightarrow S^{4}$ where $M$ has non-trivial topology. We shall see in the next section that when $f: T^{2} \rightarrow S^{4}$ is a conformally immersed 2-torus of zero normal bundle degree, there is a Riemann surface worth of Darboux transforms of $f$. There are always the trivial Darboux transforms, the constant maps $f^{\#}$, coming from holomorphic sections (without monodromy) of the 2-dimensional linear system $H \subset H^{0}(V / L)$. Whenever such a trivial Darboux transform $f^{\#}=f(p)$ is a point contained in the image of $f$, we have a singular Darboux transform and at $p \in M$ the sphere congruence $S$ degenerates to a point.

2.3. Bianchi permutability. An important feature of the classical Darboux transformation of isothermic surfaces is the following permutability property (cf. [1], [14]): if $f^{\#}$ and $f^{b}$ are Darboux transforms of an isothermic immersion $f$, then there exists an isothermic immersion $\hat{f}$ which simultaneously is a Darboux transform of $f^{\#}$ and $f^{b}$. This property carries over to general Darboux transforms of conformal immersions and will be used in Section 3 to show that the Darboux transformation is isospectral.

Theorem 2.8. Let $f: M \rightarrow S^{4}$ be a conformal immersion and let $f^{\#}, f^{b}: M \rightarrow S^{4}$ be two immersed Darboux transforms of $f$ so that $f^{\#}$ and $f^{b}$ are distinct for all points in $M$. Then there exists a conformal map $\hat{f}: M \rightarrow S^{4}$ which is a Darboux transform of $f^{\#}$ and $f^{b}$.

Proof. From Lemma 2.3 we know that there exist non-trivial sections $\psi^{\#} \in \Gamma\left(\widetilde{L^{\#}}\right)$ and $\psi^{b} \in \Gamma\left(\widetilde{L^{b}}\right)$ with monodromies $h^{\#}, h^{b}: \pi_{1}(M) \rightarrow \mathbb{H}_{*}$ satisfying $\pi d \psi^{\#}=\pi d \psi^{b}=0$. Using the splitting $V=L \oplus L^{\#}$, we obtain

$$
d \psi^{\#}=\nabla^{\#} \psi^{\#}+\delta^{\#} \psi^{\#}=\delta^{\#} \psi^{\#} \in \Gamma(\widetilde{K L}),
$$

where we again used Lemma 2.3 to see that $* \delta^{\#}=\tilde{J} \delta^{\#}$. In particular, as a parallel section $\psi^{\#}$ is nowhere vanishing and, since $f^{\#}$ is an immersion, also $d \psi^{\#}=\delta^{\#} \psi^{\#}$ is nowhere vanishing. The same argument applied to $\psi^{b}$ yields the nowhere vanishing sections $d \psi^{\#}$ and $d \psi^{b}$ with monodromies $h^{\#}$ and $h^{b}$ of the line bundle $K L$. Therefore,

$$
d \psi^{b}=d \psi^{\#} \chi
$$

where $\chi: \tilde{M} \rightarrow \mathbb{H}_{*}$ and $\gamma^{*} \chi=\left(h^{\#}\right)^{-1} \chi h^{b}$. Since $V=L^{\#} \oplus L^{b}$, the section

$$
\varphi=\psi^{b}-\psi^{\#} \chi \in \underset{\text { Bereitgestellt von | Technische Universität Berlin }}{\Gamma(\tilde{V})}
$$


with monodromy $h^{b}$ has a nowhere vanishing projection to $V / L^{\#}$. Then the line bundle $\hat{L} \subset V$ spanned by the nowhere vanishing section $\varphi$ is a Darboux transform $\hat{f}: M \rightarrow S^{4}$ of $f^{\#}$ : by Lemma 2.3 it is sufficient to show that $\pi^{\#} d \varphi=0$ which follows immediately from (2.16) and (2.15). On the other hand, the nowhere vanishing section $\varphi \chi^{-1} \in \Gamma(\tilde{\hat{L}})$ has monodromy $h^{\#}$ and exhibits $\hat{f}$ as a Darboux transform of $f^{b}$.

Remark 2.9. The proof of the previous theorem shows the following: given a conformal immersion $f: M \rightarrow S^{4}$ and an immersed Darboux transform $f^{\#}: M \rightarrow S^{4}$ of $f$, (2.16) defines a monodromy preserving map between holomorphic sections with monodromy of $V / L$ and $V / L^{\#}$.

2.4. The Willmore energy of Darboux transforms. For immersions of compact surfaces the Darboux transform preserves the Willmore energy up to topological quantities. In particular, for conformally immersed tori with trivial normal bundle the Darboux transform preserves the Willmore energy.

Lemma 2.10. Let $f: M \rightarrow S^{4}$ be a conformal immersion of a compact Riemann surface and let $f^{\#}: M \rightarrow S^{4}$ be a Darboux transform of $f$. Then

$$
\mathscr{W}\left(f^{\#}\right)=\mathscr{W}(f)+2 \pi\left(\operatorname{deg} N_{f}-\operatorname{deg} K\right),
$$

where $N_{f}=\operatorname{Hom}_{-}(L, V / L)$ is the normal bundle of $f$.

Proof. We first note that $\delta \in \Gamma\left(\operatorname{Hom}_{+}(L, K V / L)\right)$ is a holomorphic bundle isomorphism: the holomorphic structure $\left(\nabla^{L}\right)^{\prime \prime}$ on $L$ comes from the splitting $(2.10)$ and $K V / L$ has the holomorphic structure $d^{\nabla}$, where $\nabla$ is the flat adapted connection on $V / L$ given in Corollary 2.4. If $\varphi \in H^{0}(L)$, then (2.11) implies

$$
d^{\nabla} \delta \varphi=(d \delta) \varphi-\delta \wedge \nabla^{L} \varphi=-\delta \wedge\left(\nabla^{L}\right)^{\prime \prime} \varphi=0,
$$

i.e., $\delta \varphi \in H^{0}(K V / L)$, which shows that $\delta$ is a holomorphic bundle map. Since $f$ is an immersion, $\delta$ is a holomorphic isomorphism and thus $\mathscr{W}(L)=\mathscr{W}(K V / L)$. On the other hand, $\pi^{\#}$ identifies the quaternionic holomorphic bundle $V / L^{\#}$ with $L$ so that

$$
\mathscr{W}\left(V / L^{\#}\right)=\mathscr{W}(L)=\mathscr{W}(K V / L) .
$$

The adapted flat connection $\nabla$ of $V / L$ can be decomposed into $J$ commuting and anticommuting parts $\nabla=\hat{\nabla}+A+Q$, where $\hat{\nabla}=\partial+\bar{\partial}$ is a complex connection and

$$
A \in \Gamma\left(K \text { End }_{-}(V / L)\right) \text { and } Q \in \Gamma\left(K \text { End }_{-}(V / L)\right)
$$

are endomorphism valued 1-forms of type $K$ and $\bar{K}$ respectively. Since $\nabla$ is adapted, $D=\nabla^{\prime \prime}=\bar{\partial}+Q$, the Willmore energy (2.3) of $V / L$ is given by

$$
\mathscr{W}(V / L)=2 \int_{M}\langle Q \wedge * Q\rangle .
$$

Decomposing (A.4) the holomorphic structure $d^{\nabla}$ on $K V / L$ into $J$ commuting and anticommuting parts $d^{\nabla}=\bar{\partial}+\tilde{Q}$, one checks that

$$
\tilde{Q} \omega=A \wedge \omega
$$


for $\omega \in \Gamma(K V / L)$. Therefore the Willmore energy of $K V / L$ is given by

$$
\mathscr{W}(K V / L)=2 \int_{M}\langle A \wedge * A\rangle .
$$

The flatness of $\nabla=\hat{\nabla}+A+Q$ implies

$$
J \hat{R}=\langle A \wedge * A\rangle-\langle Q \wedge * Q\rangle,
$$

and (2.18), (2.19) yield

$$
4 \pi \operatorname{deg}(V / L)=\mathscr{W}(K V / L)-\mathscr{W}(V / L) .
$$

The lemma now follows from (2.17) together with the formula (2.4) for the normal bundle degree of $f$.

2.5. Conformal tori with $\int_{T^{2}}|\mathscr{H}|^{2} d \mathscr{A}<\mathbf{8} \pi$. We know from Lemma 2.1 that the sublevel set $\int_{T^{2}}|\mathscr{H}|^{2} d \mathscr{A}<8 \pi$ in the space of conformal tori $f: T^{2} \rightarrow S^{4}$ in the 4-sphere consists of conformal embeddings with trivial normal bundles. From (2.4) we see that $\operatorname{deg} V / L=0$ which implies $\mathscr{W}(f)=\int_{T^{2}}|\mathscr{H}|^{2} d \mathscr{A}$ by (2.6). Every non-constant Darboux transform $f^{\#}: T^{2} \rightarrow S^{4}$ of $f$ is again a conformal embedding and we can apply Bianchi permutability repeatedly for conformal tori in this sublevel set.

Lemma 2.11. Let $f: T^{2} \rightarrow S^{4}$ be a conformal map with $\int_{T^{2}}|\mathscr{H}|^{2} d \mathscr{A}<8 \pi$. Then

(i) $f$ is a conformal embedding and $\mathscr{W}(f)=\int_{T^{2}}|\mathscr{H}|^{2} d \mathscr{A}$,

(ii) every non-constant Darboux transform $f^{\#}: T^{2} \rightarrow S^{4}$ is a conformal embedding and $\int_{T^{2}}\left|\mathscr{H}^{\#}\right|^{2} d \mathscr{A}^{\#}=\int_{T^{2}}|\mathscr{H}|^{2} d \mathscr{A}$,

(iii) two distinct non-constant Darboux transforms $f^{\#}$ and $f^{b}$ of $f$ satisfy $f^{\#}(p) \neq f^{b}(p)$ for all $p \in T^{2}$, and of $f$.

(iv) the only singular Darboux transforms are the constant maps contained in the image

Proof. From Lemma 2.7 we see that a non-constant Darboux transform $f^{\#}$ is singular if and only if its corresponding holomorphic section $\psi \in H^{0}(\widetilde{V / L})$ with monodromy has a zero at, say, $p \in T^{2}$. Since $\operatorname{dim} H^{0}(V / L) \geqq 2$, there is always a holomorphic section $\varphi \in H^{0}(V / L)$ vanishing at $p \in T^{2}$. Therefore, the 2-dimensional linear system $H \subset H^{0}(\widetilde{V / L})$ with monodromy (A.9), (A.11) spanned by $\psi$ and $\varphi$ has ord $H \geqq 2$. But then the Plücker formula (A.10) together with $\operatorname{deg} V / L=0$ gives the contradiction $\mathscr{W}(f)=\mathscr{W}(V / L) \geqq 8 \pi$.

Therefore all non-constant Darboux transform $f^{\#}$ are non-singular so that $V=L \oplus L^{\#}$. Since $f$ is an embedding, $* \delta=J \delta=\delta \tilde{J}$, where $\delta \in \Gamma\left(K \operatorname{Hom}_{+}(L, V / L)\right)$ 
is an isomorphism, which shows that the line bundle $L$ with complex structure $\tilde{J}$ has $\operatorname{deg} L=0$. On the other hand, Lemma 2.3 says that the Darboux transform $f^{\#}$ has $* \delta^{\#}=\tilde{J} \delta^{\#}$, which implies that the line bundle $V / L^{\#}=L$ with complex structure $\tilde{J}$ has $\operatorname{deg} V / L^{\#}=0$. Applying (2.6) and Lemma 2.10, we therefore obtain

$$
\int_{T^{2}}\left|\mathscr{H}^{\#}\right|^{2} d \mathscr{A}^{\#}=\mathscr{W}\left(f^{\#}\right)=\mathscr{W}(f)=\int_{T^{2}}|\mathscr{H}|^{2} d \mathscr{A}<8 \pi
$$

which shows that also $f^{\#}$ is a conformal embedding by Lemma 2.1.

If two non-constant Darboux transforms $f^{\#}$ and $f^{b}$ of $f$ had a point in common, the section $\varphi$ in (2.16) would project under $\pi^{\#}$ to a holomorphic section with monodromy of $V / L^{\#}$ with a zero. Thus, the common non-constant Darboux transform $\hat{f}$ of $f^{\#}$ and $f^{b}$ would be singular.

Finally, if $f^{\#}$ is constant, then $V=L \oplus L^{\#}$ if and only if $f^{\#}$ is not in the image of $f$.

\section{The spectral curve}

In this section we show that a conformally immersed torus $f: T^{2} \rightarrow S^{4}$ of zero normal bundle degree gives rise to a Riemann surface, the spectral curve $\Sigma$ of $f$. Our approach is geometric because the spectral curve is introduced as the space parameterizing Darboux transforms of $f$. This point of view yields a natural $T^{2}$-family of holomorphic maps of $\Sigma$ into $\mathbb{P}^{3}$ from which the conformal immersion $f: T^{2} \rightarrow S^{4}$ can be reconstructed.

3.1. The spectrum of a holomorphic line bundle over a torus. We recall that Lemma 2.7 characterizes a Darboux transform $f^{\#}: M \rightarrow S^{4}$ of a conformal immersion $f: M \rightarrow S^{4}$ by a non-trivial holomorphic section $\psi \in \Gamma(\widetilde{V / L})$ with monodromy $h: \pi_{1}(M) \rightarrow \mathbb{H}_{*}$. Scaling this holomorphic section conjugates the representation $h$ but does not affect the Darboux transform $f^{\#}$. This suggests describing the parameter space of Darboux transforms analytically in terms of the possible monodromies $h: \pi_{1}(M) \rightarrow \mathbb{H}_{*}$ of non-trivial holomorphic sections of $\widetilde{V / L}$ up to conjugation.

Definition 3.1. Let $W$ be a quaternionic line bundle with holomorphic structure $D$ over a Riemann surface $M$. The quaternionic spectrum of $W$ is the subspace

$$
\operatorname{Spec}_{\mathbb{\sharp}}(W, D) \subset \operatorname{Hom}\left(\pi_{1}(M), \mathbb{H}_{*}\right) / \mathbb{H}_{*}
$$

of conjugacy classes of possible monodromy representations $h: \pi_{1}(M) \rightarrow \mathbb{H}_{*}$ for holomorphic sections of $\tilde{W}$. In other words, $h$ represents a point in $\operatorname{Spec}_{\square \rightarrow ~}(W, D)$ if and only if there exists a non-trivial section $\psi \in \Gamma(\tilde{W})$ with

$$
D \psi=0 \quad \text { and } \quad \gamma^{*} \psi=\psi h_{\gamma},
$$

where $\gamma \in \pi_{1}(M)$ acts by deck transformations.

Applying this notion to the holomorphic line bundle $V / L$ induced by a conformal immersion $f: M \rightarrow S^{4}$, we obtain from Lemma 2.7 a surjective map from the space of 
(singular) Darboux transforms of $f$ onto the quaternionic $\operatorname{spectrum~} \operatorname{Spec}_{\mathbb{}}(V / L, D)$ of $V / L$. Under this map the constant Darboux transforms, arising from the linear system $H \subset H^{0}(V / L)$, correspond to the trivial representation.

In what follows we confine our discussion to the case when $M=T^{2}$ is a torus. Then more can be said about the structure of the quaternionic spectrum $\operatorname{Spec}_{\mathbb{t}}(W, D)$ of a holomorphic line bundle $W$. Since $T^{2}=\mathbb{R}^{2} / \Gamma$ has abelian fundamental group $\pi_{1}\left(T^{2}\right)=\Gamma$, every representation in $\operatorname{Hom}\left(\Gamma, \mathbb{U}_{*}\right)$ can be conjugated into a complex representation in $\operatorname{Hom}\left(\Gamma, \mathbb{C}_{*}\right)$. Furthermore, conjugating a complex representation $h$ by the quaternion $j$ results in the representation $\bar{h} \in \operatorname{Hom}\left(\Gamma, \mathbb{C}_{*}\right)$. Therefore, the map

$$
\operatorname{Hom}\left(\Gamma, \mathbb{C}_{*}\right) \rightarrow \operatorname{Hom}\left(\Gamma, \mathbb{M}_{*}\right) / \mathbb{W}_{*}
$$

is $2: 1$ away from real representations and the quaternionic spectrum $\operatorname{Spec}_{\mathbb{t}}(W, D)$ of $W$ lifts to the complex spectrum

$$
\operatorname{Spec}(W, D) \subset \operatorname{Hom}\left(\Gamma, \mathbb{C}_{*}\right)
$$

given by the possible complex monodromies for holomorphic sections of $\tilde{W}$. By construction, the complex spectrum is invariant under complex conjugation $\rho(h)=\bar{h}$ and the quaternionic spectrum

$$
\operatorname{Spec}_{\mathbb{1}}(W, D)=\operatorname{Spec}(W, D) / \rho
$$

is the quotient of the complex spectrum under $\rho$.

The abelian complex Lie group $\operatorname{Hom}\left(\Gamma, \mathbb{C}_{*}\right)$ has as Lie algebra $\operatorname{Hom}(\Gamma, \mathbb{C})$ which, via the period map, is isomorphic to the vector space of harmonic 1-forms $\operatorname{Harm}\left(T^{2}, \mathbb{C}\right)$ on the torus $T^{2}$. The exponential map

$$
\exp : \operatorname{Harm}\left(T^{2}, \mathbb{C}\right) \rightarrow \operatorname{Hom}\left(\Gamma, \mathbb{C}_{*}\right) \quad \text { defined by } \exp (\omega)=e^{\int \omega}
$$

has the integer harmonic forms $\Gamma^{*}=\operatorname{Harm}\left(T^{2}, 2 \pi i \mathbb{Z}\right)$ as its kernel and thus induces the holomorphic isomorphism

$$
\operatorname{Harm}\left(T^{2}, \mathbb{C}\right) / \Gamma^{*} \cong \operatorname{Hom}\left(\Gamma, \mathbb{C}_{*}\right)
$$

In order to see that the spectrum is an analytic variety, we lift the spectrum to the $\Gamma^{*}$-periodic logarithmic spectrum

$$
\widetilde{\operatorname{Spec}}(W, D)=\exp ^{-1}(\operatorname{Spec}(W, D)) \subset \operatorname{Harm}\left(T^{2}, \mathbb{C}\right)
$$

which consists of harmonic forms $\omega$ for which there is a holomorphic section $\psi \in H^{0}(\tilde{W})$ with monodromy $h=e^{\int \omega}$. Interpreting $e^{\int \omega} \in \operatorname{Hom}\left(\mathbb{R}^{2}, \mathbb{C}_{*}\right)$ as a (non-periodic) gauge transformation, we see that the section $\psi e^{-\int \omega} \in \Gamma(W)$ has trivial monodromy and lies in the kernel of the gauged operator

$$
D_{\omega}=e^{-\int \omega} \circ D \circ e^{\int \omega}: \Gamma(W) \rightarrow \Gamma(\bar{K} W) .
$$


The operator $D_{\omega}$ is defined on the torus (even though the gauge is not) because the Leibniz rule (A.3) of a quaternionic holomorphic structure implies

$$
D_{\omega}(\psi)=D \psi+(\psi \omega)^{\prime \prime}
$$

and both $D$ and $\omega$ are defined on the torus $T^{2}$. Moreover, $D_{\omega}$ is elliptic and, due to the term $(\psi \omega)^{\prime \prime}$ in (3.6), complex linear (rather than quaternionic linear) between the complex rank 2 bundles $W$ and $\bar{K} W$ whose complex structures are given by right multiplication by the quaternion $i$. Thus, the gauge transformation $e^{\int \omega}$ induces the complex linear isomorphism

$$
\operatorname{ker} D_{\omega} \rightarrow H_{h}^{0}(\tilde{W}), \quad \psi \mapsto \psi e^{\int \omega},
$$

where $H_{h}^{0}(\tilde{W})$ denotes the complex vector space of holomorphic sections of $W$ with monodromy $h=e^{\int \omega}$. The logarithmic spectrum therefore is the locus of harmonic forms

$$
\widetilde{\operatorname{Spec}}(W, D)=\left\{\omega \in \operatorname{Harm}\left(T^{2}, \mathbb{C}\right) \mid \operatorname{ker} D_{\omega} \neq 0\right\} \subset \operatorname{Harm}\left(T^{2}, \mathbb{C}\right)
$$

for which $D_{\omega}$ has a non-trivial kernel. But $D_{\omega}$ is a holomorphic family of elliptic operators over $\operatorname{Harm}\left(T^{2}, \mathbb{C}\right)$, which implies that the logarithmic spectrum $\widetilde{\operatorname{Spec}}(W, D)$, and hence also the spectrum $\operatorname{Spec}(W, D)=\widetilde{\operatorname{Spec}}(W, D) / \Gamma^{*}$ as a quotient by $\Gamma^{*}$, are analytic varieties in $\operatorname{Harm}\left(T^{2}, \mathbb{C}\right)$ and $\operatorname{Hom}\left(\Gamma, \mathbb{C}_{*}\right)$ respectively.

3.2. Homogeneous bundles. At this stage it is instructive to discuss an explicit example, namely the spectrum of a homogeneous torus in $S^{4}$. Such a conformally immersed torus $f: T^{2} \rightarrow S^{4}$ is a $T^{2}$-orbit of the Möbius group and hence a product of two circles in perpendicular planes in $S^{3}$. The induced holomorphic line bundle $V / L$ is therefore invariant under translations by $T^{2}$ and has zero degree. More generally, we call a quaternionic holomorphic line bundle $W$ over a torus $T^{2}$ homogeneous if for each $a \in T^{2}$ there is a holomorphic bundle isomorphism $T_{a}: W \rightarrow a^{*} W$. Such bundles always have degree zero. What makes it possible to explicitly compute the spectrum of a homogeneous line bundle is the fact that the holomorphic structure $D=\bar{\partial}+Q$ has constant $Q$ in an appropriate trivialization.

Lemma 3.2. Let $W$ be a quaternionic line bundle over a torus $T^{2}$ with holomorphic structure $D=\bar{\partial}+Q$. If $Q \neq 0$, then $W$ is homogeneous if and only if $W$ is, up to tensoring by a $\mathbb{Z}^{2}$-bundle, holomorphically isomorphic to the trivial $\mathbb{C}^{2}$-bundle with holomorphic structure

$$
\hat{D}=\left(\begin{array}{cc}
\hat{\bar{\partial}} & -\overline{\hat{q}} \\
\hat{q} & \hat{\partial}
\end{array}\right),
$$

where $\hat{q} \in H^{0}(K)$ is "constant". The Willmore integrand of the holomorphic structure $D$ is given by $\langle Q \wedge * Q\rangle=i \hat{q} \wedge \overline{\hat{q}}$.

Proof. Let $J \in \Gamma(\operatorname{End}(W))$ denote the complex structure of $W$. The $i$-eigenspace $W_{+}$of $J$ is a degree zero complex line bundle with holomorphic structure $\bar{\partial}$. Since $W_{+}$has zero degree, $\bar{\partial}=\bar{\partial}_{0}-\bar{\alpha}$ with $\alpha \in H^{0}(K)$ a holomorphic 1-form and $\bar{\partial}_{0}$ a trivial holomorphic structure. Let $\psi \in \Gamma\left(W_{+}\right)$be a holomorphic trivialization with respect to $\bar{\partial}_{0}$, that is, 
$\bar{\partial}_{0} \psi=0$. In this trivialization,

$$
Q \psi=\psi j q
$$

for some non-zero $q \in \Gamma(K)$ since $Q$ anticommutes with $J$. For each $a \in T^{2}$ the holomorphic bundle isomorphism $T_{a}$ intertwines the complex and holomorphic structures on $W$ and $a^{*} W$, namely

$$
\left(a^{*} J\right) T_{a}=T_{a} J, \quad\left(a^{*} \bar{\partial}\right) T_{a}=T_{a} \bar{\partial}, \quad\left(a^{*} Q\right) T_{a}=T_{a} Q .
$$

Evaluating these conditions on the trivializing section $\psi$ gives $T_{a} \psi=\left(a^{*} \psi\right) \lambda_{a}$ with $\lambda_{a} \in \mathbb{C}$ and

$$
a^{*} q=u_{a}^{2} q
$$

where $u_{a}$ is the unitary part of $\overline{\lambda_{a}}$. This implies that $u^{2}: T^{2} \rightarrow S^{1}$ is a representation if $q$ is non-zero, and hence $u^{2}=e^{\int \eta}$ with $\eta \in \Gamma^{*}$ an integer period harmonic form. Let $R$ be the flat real bundle defined by the representation $e^{\frac{1}{2} \int \eta}: \Gamma \rightarrow \mathbb{Z}_{2}$ and denote by $\varphi$ a parallel section of $R$ with monodromy $e^{\frac{1}{2} \int \eta}$. Viewing $e^{\frac{1}{2} \int \eta}$ as a function on the universal cover $\mathbb{R}^{2}$, we see that $\hat{\psi}=\psi \otimes \phi e^{-\frac{1}{2} \int \eta}$ is a trivializing section of $W \otimes R$. By construction, $\hat{\psi} \in \Gamma\left((W \otimes R)_{+}\right)$ and

$$
\hat{\bar{\partial}} \hat{\psi}=\hat{\psi}\left(\bar{\alpha}-\frac{1}{2} \eta^{\prime \prime}\right)=\hat{\psi} \overline{\hat{\alpha}}
$$

with $\hat{\alpha} \in H^{0}(K)$. Moreover, $\hat{Q}$ is constant in this trivialization since

$$
\hat{Q} \hat{\psi}=(Q \psi) \otimes \phi e^{-\frac{1}{2} \int \eta}=\psi j q \otimes \phi e^{-\frac{1}{2} \int \eta}=\hat{\psi} j e^{-\int \eta} q
$$

and by (3.9) the $(1,0)$-form $\hat{q}=e^{-\int \eta} q$ is translation invariant and thus holomorphic. The form of $\hat{D}$ now follows from trivializing $W \otimes R$ using the frame $\psi, \psi j$ and (A.7).

We are now in a position to calculate the spectrum of a homogeneous bundle $W$ : tensoring by the $\mathbb{Z}_{2}$-bundle given by the representation $e^{-\frac{1}{2} \int \eta_{0}}$ for $\eta_{0} \in \Gamma^{*}$ relates the spectra of $D$ and $\hat{D}$ by this representation. In particular, the logarithmic spectra

$$
\widetilde{\operatorname{Spec}}(D)=\frac{1}{2} \eta_{0}+\widetilde{\operatorname{Spec}}(\hat{D})
$$

are related via a shift by the half lattice vector $\frac{1}{2} \eta_{0}$. Ignoring this shift for the moment, we may assume that the holomorphic structure $D$ on $W$ already has constant $Q$ and $D$ is given as in the lemma. Moreover, replacing the complex holomorphic structure $\bar{\partial}=\bar{\partial}_{0}-\bar{\alpha}$ in $D=\bar{\partial}+Q$, where $\alpha \in H^{0}(K)$, by the trivial holomorphic structure $\bar{\partial}_{0}$ results in an additional shift of $\widehat{\operatorname{Spec}}(D)$ by $\alpha+\bar{\alpha}$. Thus, we may assume that $\bar{\partial}$ is already trivial. The holomorphic family of elliptic operators $D_{\omega}$, parameterized by $\omega \in \operatorname{Harm}\left(T^{2}, \mathbb{C}\right)$, then has the form

$$
D_{\omega}=\left(\begin{array}{cc}
\bar{\partial}+\omega^{\prime \prime} & -\bar{q} \\
q & \partial+\omega^{\prime}
\end{array}\right)
$$


with $q \in H^{0}(K)$. Therefore $D_{\omega}$ commutes with translations of the torus $T^{2}$ so that the finite dimensional kernel of $D_{\omega}$ is spanned by eigenlines of the translation operators on $\Gamma\left(\underline{\mathbb{C}}^{2}\right)$. These are given by the Fourier modes $\psi_{\eta}=v e^{\int \eta}$ with $v \in \mathbb{C}^{2}$ and $\eta \in \Gamma^{*}$. From

$$
D_{\omega} \psi_{\eta}=\left(\begin{array}{cc}
(\eta+\omega)^{\prime \prime} & -\bar{q} \\
q & (\eta+\omega)^{\prime}
\end{array}\right) \psi_{\eta}
$$

we see that $\omega \in \widetilde{\operatorname{Spec}}(D)$ if and only if there exists $\eta \in \Gamma^{*}$ such that

$$
(\omega+\eta)^{\prime}(\omega+\eta)^{\prime \prime}+|q|^{2}=0
$$

We first discuss the vacuum spectrum, that is, $Q=0$. In this case the logarithmic spectrum

$$
\widetilde{\operatorname{Spec}}(\bar{\partial})=\left\{\omega \in \operatorname{Harm}\left(T^{2}, \mathbb{C}\right) \mid \omega^{\prime}=\eta^{\prime} \text { or } \omega^{\prime \prime}=\eta^{\prime \prime} \text { for } \eta \in \Gamma^{*}\right\}
$$

consists of $\Gamma^{*}$-translates of the complex lines $H^{0}(K)$ and $\overline{H^{0}(K)}$ in $\operatorname{Harm}\left(T^{2}, \mathbb{C}\right)$. Therefore, the vacuum spectrum $\operatorname{Spec}(\bar{\partial}) \subset \operatorname{Hom}\left(\Gamma, \mathbb{C}_{*}\right)$ is the union

$$
\operatorname{Spec}(\bar{\partial})=\exp \left(H^{0}(K)\right) \cup \exp \left(\overline{H^{0}(K)}\right)
$$

which is a singular curve with double points at the lattice of real representations. The normalization $\Sigma_{0}$ of the vacuum spectrum is disconnected and consists of two copies of $\mathbb{C}$ which are exchanged under the real structure $\rho$. By adding two points "at infinity", $\Sigma_{0}$ can be compactified to two copies of $\mathbb{P}^{1}$. From (3.10) we see that the kernel ker $\bar{\partial}_{\omega}$ for $\omega \in \widehat{\operatorname{Spec}}(\bar{\partial})$ is spanned by $\left(0, e^{\int \eta}\right)$ or $\left(e^{\int \eta}, 0\right)$, depending on whether $\omega^{\prime}=-\eta^{\prime}$ or $\omega^{\prime \prime}=-\eta^{\prime \prime}$. Thus, $\operatorname{ker} \bar{\partial}_{\omega}$ for $\omega \in \widehat{\operatorname{Spec}}(\bar{\partial})$ is 1-dimensional except for double points of the spectrum, where it is 2-dimensional.

If $Q \neq 0$, then (3.11) shows that the logarithmic spectrum $\widetilde{\operatorname{Spec}}(D)$ is a $\Gamma^{*}$-periodic union of conics which are asymptotic to the logarithmic vacuum spectrum $\widehat{\operatorname{Spec}}(\bar{\partial})$. From (3.11) we also see that the double point at the trivial representation in the spectrum $\operatorname{Spec}(\bar{\partial})$ is resolved into a handle in the spectrum $\operatorname{Spec}(D)$. This has the effect that the normalization $\Sigma$ of the spectrum $\operatorname{Spec}(D)$ is connected with two ends asymptotic to $\exp \left(H^{0}(K)\right)$ and $\exp \left(\overline{H^{0}(K)}\right)$ respectively. Thus, $\Sigma$ can be compactified to $\mathbb{P}^{1}$ by adding two points at infinity which are interchanged by the real structure $\rho$. Moreover, from (3.10) we can calculate that the kernel of $D_{\omega}$ is generically 1-dimensional and approaches the vacuum kernel ker $\bar{\partial}_{\omega}$ near the ends of $\Sigma$.

We conclude this discussion with the case that the homogeneous bundle $W$ has a 2-dimensional linear system $H \subset H^{0}(W)$, which is certainly the case when $W$ is the induced bundle $V / L$ from a homogeneous torus $f: T^{2} \rightarrow S^{3}$. Then one can show that the complex holomorphic structure $\widehat{\bar{\partial}}$ is necessarily trivial. The half lattice vector $\frac{1}{2} \eta_{0}$ which shifts the spectrum of $\hat{D}$ to that of the homogeneous torus $f: T^{2} \rightarrow S^{3}$ is its induced spin structure. Since the holomorphic structure on $V / L$ has sections without monodromy, the trivial representation has to be a point of the spectrum $\operatorname{Spec}(V / L)$. From (3.11) we see that $|q|^{2}$ has to be bounded below by the minimum of $\left|\frac{1}{2} \eta_{0}+\eta\right|^{2}$ for $\eta \in \Gamma^{*}$. This lower 
bound on the length of $|q|^{2}$ translates into a lower bound on the Willmore energy $\mathscr{W}(f)$ of the homogeneous torus depending on its conformal and induced spin structures, attaining its minimum $2 \pi^{2}$ at the Clifford torus.

3.3. The spectral curve and the kernel bundle. The behavior of the spectrum of a homogeneous line bundle is in many ways reminiscent of the general case. The following theorem collects some of the results about the structure of the spectrum of a quaternionic holomorphic line bundle needed in the present paper and proven in [4]. We may assume the line bundle to have zero degree since otherwise its spectrum is finite or all of $\operatorname{Hom}\left(\Gamma, \mathbb{C}_{*}\right)$.

Theorem 3.3. Let $W$ be a quaternionic line bundle of degree zero over a torus $T^{2}$ with holomorphic structure $D=\bar{\partial}+Q$.

(i) The spectrum $\operatorname{Spec}(W, D)$ is a 1-dimensional analytic variety in $\operatorname{Hom}\left(\Gamma, \mathbb{C}_{*}\right)$ invariant under the real structure $\rho(h)=\bar{h}$.

(ii) The spectrum $\operatorname{Spec}(W, D)$ is asymptotic to the vacuum spectrum $\operatorname{Spec}(W, \bar{\partial})$ : outside a sufficiently large compact set in $\operatorname{Hom}\left(\Gamma, \mathbb{C}_{*}\right)$ the spectrum $\operatorname{Spec}(W, D)$ is contained in an arbitrarily small tube around $\operatorname{Spec}(W, \bar{\partial})$. Away from the double points of the vacuum spectrum outside this compact set the spectrum is a graph over the vacuum spectrum. Near the double points of the vacuum spectrum outside this compact set the spectrum is a smooth annulus or two discs intersecting in a double point.

(iii) For $h=e^{\int \omega} \in \operatorname{Spec}(W, D)$ the operator $D_{\omega}$ generically has a 1-dimensional kernel, that is, $\operatorname{dim} H_{h}^{0}(\tilde{W})=1$ away from a discrete set in $\operatorname{Spec}(W, D)$.

Normalizing the spectrum, we obtain a Riemann surface, the spectral curve of the quaternionic holomorphic line bundle.

Definition 3.4. Let $W$ be a quaternionic line bundle of degree zero over a torus $T^{2}$ with holomorphic structure $D=\bar{\partial}+Q$. The spectral curve $\Sigma$ of $W$ is the normalization $h: \Sigma \rightarrow \operatorname{Spec}(W, D)$ of the spectrum $\operatorname{Spec}(W, D)$ to a (not necessarily connected) Riemann surface.

The logarithmic spectral curve is the Riemann surface $\tilde{\Sigma}$ normalizing the logarithmic spectrum $\omega: \tilde{\Sigma} \rightarrow \widetilde{\operatorname{Spec}}(W, D)$. By the universal property of the normalization, $\tilde{\Sigma}$ is a $\Gamma^{*}$-periodic Riemann surface whose quotient under the lattice $\Gamma^{*}$ is $\Sigma=\tilde{\Sigma} / \Gamma^{*}$.

The vacuum spectral curve $\Sigma_{0}$ is the Riemann surface which normalizes the spectrum $\operatorname{Spec}(W, \bar{\partial})$ of the complex holomorphic structure $\bar{\partial}$.

The normalization map $\omega: \tilde{\Sigma} \rightarrow \widetilde{\operatorname{Spec}}(W, D)$ in Definition 3.4 pulls back the holomorphic family $D_{\omega}: \Gamma(W) \rightarrow \Gamma(\bar{K} W)$ to a holomorphic family of elliptic operators over the Riemann surface $\tilde{\Sigma}$. Thus, by Theorem 3.3 the kernels of $D_{\omega}$ give rise to a holomorphic line bundle

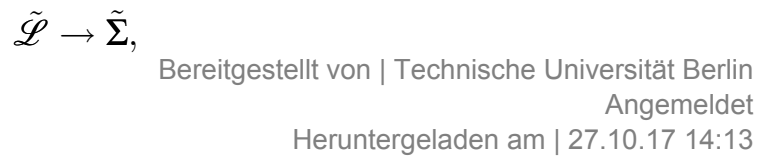


a subbundle of the trivial $\Gamma(W)$-bundle over $\tilde{\Sigma}$ (for $\Gamma(W)$ equipped with the $C^{\infty}$-topology, see [4], Lemma 2.4): the fibers $\tilde{\mathscr{L}}_{\tilde{x}}=\operatorname{ker} D_{\omega(\tilde{x})}$ coincide with the kernels of $D_{\omega(\tilde{x})}$ away from the discrete set of points $\tilde{x} \in \tilde{\Sigma}$ where the kernel dimension is greater than one. Moreover, on this discrete set $\tilde{\mathscr{L}}_{\tilde{x}} \subset \operatorname{ker} D_{\omega(\tilde{x})}$.

We have seen in (3.7) that $\operatorname{ker} D_{\omega}$ is isomorphic to $H_{h}^{0}(\tilde{W})$ where $h=e^{\int \omega}$. Since the spectral curve $\Sigma=\tilde{\Sigma} / \Gamma^{*}$ is the quotient under the action of the lattice $\Gamma^{*}$ of integer harmonic forms, the bundle $\tilde{\mathscr{L}}$ descends to a holomorphic line subbundle

$$
\mathscr{L} \rightarrow \Sigma, \quad \mathscr{L}_{x}=\tilde{\mathscr{L}}_{\tilde{x}} e^{\int \omega(\tilde{x})}
$$

of the trivial $\Gamma(\tilde{W})$-bundle over $\Sigma$, where $x=\tilde{x}+\Gamma^{*}$. Therefore, the fiber $\mathscr{L}_{x} \subset H_{h(x)}^{0}(\tilde{W})$ of $\mathscr{L}$ over $x \in \Sigma$ consists of holomorphic sections of $W$ with monodromy $h(x)$ and $\mathscr{L}_{x}=H_{h(x)}^{0}(\tilde{W})$ away from a discrete set in $\Sigma$.

Definition 3.5. Let $W$ be a quaternionic holomorphic line bundle of degree zero over a torus $T^{2}$ with spectral curve $\Sigma$. The complex holomorphic line bundle $\mathscr{L}$ over $\Sigma$ given in (3.13) with generic fiber $\mathscr{L}_{x}=H_{h(x)}^{0}(\tilde{W})$ over $x \in \Sigma$ is called the kernel bundle.

The real structure $\rho$ on $\Sigma$ acts on the kernel bundle via multiplication by the quaternion $j$ : given a holomorphic section $\psi \in H_{h(x)}^{0}(\tilde{W})$, the section $\psi j$ has monodromy $\overline{h(x)}=h(\rho(x))$. Therefore, the real structure $\rho$ is fixed point free and the kernel bundle is compatible with the real structure, that is, $\rho^{*} \mathscr{L}=\mathscr{L} j$. In particular, $\Sigma / \rho$ normalizes $\operatorname{Spec}_{\mathbb{H}}(W, D)$ to a non-orientable (if $\Sigma$ is connected) Riemann surface by (3.1).

From Theorem 3.3 and the fact that $\Sigma$ cannot have compact components, we see that $\Sigma$ has one or two ends and at most two components each of which contains an end and which are exchanged under $\rho$. In case $\Sigma$ has one end, $\Sigma$ is connected and the genus of $\Sigma$ is necessarily infinite. In the finite genus case, $\Sigma$ has two ends. We summarize the discussion so far:

Theorem 3.6. Let $W$ be a quaternionic holomorphic line bundle of degree zero over a torus $T^{2}$. Then the spectral curve $\Sigma$ is a Riemann surface with a fixed point free real structure $\rho$, one or two ends and at most two components each of which contains an end. Depending on whether $\Sigma$ has one or two ends, the genus of $\Sigma$ is infinite and $\Sigma$ is connected, or the genus of $\Sigma$ is finite. The kernel bundle $\mathscr{L}$ is a complex holomorphic line bundle over $\Sigma$ compatible with the real structure, that is, $\rho^{*} \mathscr{L}=\mathscr{L} j$.

We now return to the case of interest to us when the quaternionic holomorphic line bundle $W$ is the line bundle $V / L$ induced by a conformal immersion $f: T^{2} \rightarrow S^{4}$ of zero normal bundle degree. In this situation the fibers of the kernel bundle $\mathscr{L}$ have a geometric interpretation in terms of Darboux transformations. By Definition 3.5, the fiber $\mathscr{L}_{x} \subset H_{h(x)}^{0}(\widetilde{V / L})$ of $\mathscr{L}$ over $x \in \Sigma$ consists of a complex line worth of non-trivial sections $\psi$ of $\widehat{V / L}$ with monodromy $h(x) \in \operatorname{Hom}\left(\Gamma, \mathbb{C}_{*}\right)$. As we have seen in Lemma 2.5 such a $\psi$ can be prolonged to sections $\hat{\psi} \in \Gamma_{h(x)}(\tilde{V})$ with the same monodromy $h(x)$. Therefore, we have constructed a complex holomorphic line subbundle

$$
\hat{\mathscr{L}} \rightarrow \Sigma
$$


of the trivial $\Gamma(\tilde{V})$-bundle over the spectral curve $\Sigma$. Moreover, Lemma 2.7 shows that

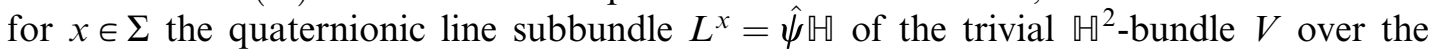
torus $T^{2}$ with $\hat{\psi} \in \hat{\mathscr{L}}_{x}$ is a (possibly singular) Darboux transform

$$
f^{x}: T^{2} \rightarrow S^{4}
$$

of $f$. On the other hand, evaluating the holomorphic line subbundle $\hat{\mathscr{L}}$ of the trivial $\Gamma(\tilde{V})$ bundle for fixed $p \in T^{2}$ gives the holomorphic map

$$
\hat{F}(p,-): \Sigma \rightarrow \mathbb{P}^{3} \quad \text { defined by } \hat{F}(p,-)=\hat{\mathscr{L}}(p) \subset \mathbb{H}^{2} .
$$

From the invariance of the kernel bundle we see that $\rho^{*} \hat{F}(p,-)=\hat{F}(p,-) j$. This implies that under twistor projection $\mathbb{C P}^{3} \rightarrow \mathbb{U} \mathbb{P}^{1}$ the map $\hat{F}(p,-)$ induces the conformal map

$$
F(p,-): \Sigma \rightarrow \mathbb{H} \mathbb{P}^{1}, \quad F(p, x)=f^{x}(p)
$$

realizing the spectral curve $\Sigma$, in fact its quotient $\Sigma / \rho$ under the real structure $\rho$, as the twistor projection into $S^{4}$ of a holomorphic curve in $\mathbb{P}^{3}$, that is, as a super conformal Willmore surface.

Generally, the $T^{2}$-family $\hat{F}(p,-)$ of holomorphic curves in $\mathbb{P}^{3}$ will not be smooth in $p \in T^{2}$ : second and higher order zeros on $T^{2}$ of sections $\psi \in \mathscr{L}_{x}$ give rise to zeros of the prolonged section $\hat{\psi} \in \Gamma(\tilde{V})$, which can cause bubbling off phenomena.

The situation simplifies considerably under the assumption that the conformal immersion $f: T^{2} \rightarrow S^{4}$ has $\mathscr{W}(f)<8 \pi$. Then Lemma 2.11 ensures that the sections in $\hat{\mathscr{L}}_{x}$ for $x \in \Sigma$ have no zeros and every non-constant Darboux transform $f^{x}$ is a conformal embedding.

Theorem 3.7. Let $f: T^{2} \rightarrow S^{4}$ be a conformal immersion with trivial normal bundle, induced holomorphic line bundle $V / L$, and spectral curve $\Sigma$. Then there exists a map

$$
F: T^{2} \times \Sigma \rightarrow S^{4}
$$

with the following properties:

(i) For $x \in \Sigma$ the map $f^{x}=F(-, x): T^{2} \rightarrow S^{4}$ is a (possibly singular) Darboux transform of $f$. In the non-singular case its Willmore energy is $\mathscr{W}\left(f^{x}\right)=\mathscr{W}(f)$. If the Willmore energy $\mathscr{W}(f)<8 \pi$, then each non-constant Darboux transform $f^{x}$ is a conformal embedding.

(ii) For $p \in T^{2}$ the map $F(p,-): \Sigma \rightarrow S^{4}$ is the twistor projection of a holomorphic curve $\hat{F}(p,-): \Sigma \rightarrow \mathbb{P}^{3}$ equivariant with respect to the real structure $\rho$ on $\Sigma$ and multiplication by $j$ on $\mathbb{P}^{3}=\mathbb{P}\left(\mathbb{M}^{2}\right)$.

(iii) If the Willmore energy of $f$ satisfies $\mathscr{W}(f)<8 \pi$, then $\hat{F}: T^{2} \times \Sigma \rightarrow \mathbb{P}^{3}$ is a smooth map which is conformal in the first and holomorphic in the second factor. 
We conclude this section with a fact which lies at the heart of the integrable systems approach to conformal maps of 2-tori into the 4-sphere: the spectral curve is a first integral of the discrete flow given by Darboux transforms on the space of conformal tori in $S^{4}$ with zero normal bundle degree.

Theorem 3.8. Let $f, f^{\#}: T^{2} \rightarrow S^{4}$ be conformal immersions so that $f^{\#}$ is a Darboux transform of $f$. Then the spectral curves of $f$ and $f^{\#}$ agree, that is, $\Sigma=\Sigma^{\#}$.

Proof. From Remark 2.9 we know that $\operatorname{Spec}(V / L) \subset \operatorname{Spec}\left(V / L^{\#}\right)$. But the spectrum is an analytic curve in $\operatorname{Harm}\left(T^{2}, \mathbb{C}_{*}\right)$ asymptotic to the vacuum spectrum by Theorem 3.3. This implies that the spectra of $V / L$ and $V / L^{\#}$ have to agree.

\section{Conformal 2-tori in $S^{4}$ of finite spectral genus}

At this stage we have constructed to each conformal immersion $f: T^{2} \rightarrow S^{4}$ of a 2-torus into the 4-sphere with trivial normal bundle a Riemann surface, the spectral curve $\Sigma$, of possibly infinite genus, with one or two ends and a fixed point free real structure $\rho$. This curve carries a complex holomorphic line bundle $\hat{\mathscr{L}} \rightarrow \Sigma$, the prolongation of the kernel bundle $\mathscr{L}$, which gives rise to Darboux transforms of $f$. The line bundle is compatible with the real structure in the sense that $\rho^{*} \hat{\mathscr{L}}=\hat{\mathscr{L}}$. For these "spectral data" to be of algebro-geometric nature, the curve $\Sigma$ has to complete to a compact Riemann surface and the line bundle $\hat{\mathscr{L}}$ has to extend holomorphically to the compactification. For the generic conformal immersion $f$ this will not be possible. But there are interesting examples of conformal immersions, including constant mean curvature (cf. [21], [15]) and (constrained) Willmore tori (cf. [22], [3]), for which the spectral data become algebro-geometric due to the ellipticity of the underlying variational problems. From the asymptotic behavior of the spectral curve $\Sigma$ outlined in Theorem 3.3, we know that away from handles each end of $\Sigma$ is asymptotic to a plane. Thus, if $\Sigma$ has finite genus, and therefore must have two ends, it can be compactified by adding two points $o$ and $\rho(o)=\infty$ at the ends.

Definition 4.1. A conformal immersion $f: T^{2} \rightarrow S^{4}$ has finite spectral genus if its spectral curve $\Sigma$ has finite genus. In this case $\Sigma$ has two ends and can be completed to a compact Riemann surface $\bar{\Sigma}$ by adding two points $o$ and $\rho(o)=\infty$ at the ends.

In the mathematical physics literature also the term "finite gap" is used: asymptotically, $\operatorname{Spec}(V / L, D)$ with $D=\bar{\partial}+Q$ lies near the vacuum $\operatorname{spectrum} \operatorname{Spec}(V / L, \bar{\partial})$, which has a lattice of double points. Generically, $\operatorname{Spec}(V / L, D)$ resolves all the double points into handles, but in the finite spectral genus case only finitely many handles appear, and the ends can be compactified by adding two points.

We now show that the $T^{2}$-family of holomorphic curves from $\Sigma$ to $\mathbb{P}^{3}$ given by the prolonged kernel bundle $\hat{\mathscr{L}}$ extends holomorphically to the compactified spectral curve $\bar{\Sigma}=\Sigma \cup\{o, \infty\}$.

Theorem 4.2. Let $f: T^{2} \rightarrow S^{4}$ be a conformal immersion with trivial normal bundle whose spectral curve $\Sigma$ has finite genus. As in Theorem 3.7 we denote by $\hat{F}: T^{2} \times \Sigma \rightarrow \mathbb{P}^{3}$ the $T^{2}$-family of holomorphic curves with twistor projection $F: T^{2} \times \Sigma \rightarrow S^{4}$. Then $\hat{F}$ extends to the compactification $\hat{F}: T^{2} \times \bar{\Sigma} \rightarrow \mathbb{P}^{3}$ and satisfies: 
(i) $\hat{F}(p,-): \bar{\Sigma} \rightarrow \mathbb{P}^{3}$ is an algebraic curve for each $p \in T^{2}$.

(ii) The original conformal immersion $f: T^{2} \rightarrow S^{4}$ is obtained by evaluation

$$
f=F(-, o)=F(-, \infty)
$$

of the $T^{2}$-family at the points at infinity.

(iii) If the Willmore energy satisfies $\mathscr{W}(f)<8 \pi$, then the map $\hat{F}: T^{2} \times \bar{\Sigma} \rightarrow \mathbb{P}^{3}$ is a smooth $T^{2}$-family of algebraic curves.

The proof of this theorem is based on the following result from [4]:

Lemma 4.3. Let $W$ be a quaternionic line bundle of degree zero over a torus $T^{2}$ with complex structure $J$ and holomorphic structure $D$ whose spectral curve $\Sigma$ has finite genus.

(i) Outside a sufficiently large compact set $K \subset \Sigma$ the fibers $\mathscr{L}_{x}, x \in K$, of the eigenline bundle $\mathscr{L}$ are spanned by a holomorphic family of nowhere vanishing holomorphic sections $\psi^{x} \in H_{h(x)}^{0}(\tilde{W})$.

(ii) The holomorphic family of complex structures $S^{x}$ on $W$ given by $S^{x} \psi^{x}=\psi^{x} i$, $x \in K$, extends holomorphically to $\bar{\Sigma} \backslash K$ with $S^{o}=J$ and $S^{\infty}=-J$.

Applying this lemma to the bundle $V / L$ induced by a conformal immersion is a key ingredient of the proof of the above theorem.

Proof. We have to show that for given $p \in T^{2}$ the holomorphic curve $\hat{F}(p,-): \Sigma \rightarrow \mathbb{P}^{3}$ extends to the compactification $\bar{\Sigma}$. Let $L_{0}$ be a fixed Darboux transform of $f$ such that $V=L \oplus L_{0}$. From Lemma 4.3 we know that for $x \in \Sigma$ near an end the fiber $\hat{\mathscr{L}}_{x}=\hat{\psi}^{x} \mathbb{C}$ of the prolonged kernel bundle $\hat{\mathscr{L}}$ is spanned by the prolongation $\widehat{\psi^{x}}$ of a nowhere vanishing holomorphic section $\psi^{x} \in H_{h(x)}^{0}(\widetilde{V / L})$ with monodromy $h(x)$. Thus, we have a Darboux transform $L^{x}=\hat{\psi}^{x} \boxplus \subset V$ for such $x \in \Sigma$. Since $\psi^{x}$ is holomorphic, the connection $\nabla^{x}$ on $V / L$ rendering $\psi^{x}$ parallel is adapted, that is, $\left(\nabla^{x}\right)^{\prime \prime}=D$ is the holomorphic structure induced by $f$ on $V / L$. Moreover, by Lemma 4.3 the holomorphic family of complex structures $S^{x}$ given by $S^{x} \psi^{x}=\psi^{x} i$ extends holomorphically to the compactification $\bar{\Sigma}$ with $S^{\infty}=J$ and $S^{o}=-J$, where $J$ is the complex structure $(2.1)$ on $V / L$ coming from the immersion $f$.

We express $L^{x}=\left(1+R^{x}\right) L_{0}$ as a graph, where $R^{x} \in \Gamma(\operatorname{Hom}(V / L, L))$. Then the adapted connection $\nabla^{x}$ is given by

$$
\nabla^{x}=\nabla_{0}+\delta R^{x}
$$

where $\nabla_{0}$ is the adapted connection on $V / L$ induced by the splitting $V=L \oplus L_{0}$ from Corollary 2.4. Denote by $\hat{\nabla}$ the unique flat connection on $V / L=E_{0} \oplus E_{0}$ compatible with $J, \bar{\partial}$, and with unitary monodromy, where $E_{0}$ is the $i$-eigenspace of $J$ on $V / L$. Then

$$
\nabla^{x}=\hat{\nabla}+\eta^{x} \quad \text { and } \quad \nabla_{0}=\hat{\nabla}+\eta_{0}
$$


and we have

$$
\eta^{x}=\eta_{0}+\delta R^{x}
$$

Now let $E^{x} \subset V / L \cong L_{0}$ denote the $i$-eigenspace of $S^{x}$. Then $\left(1+R^{x}\right) E^{x}=\widehat{\psi^{x}} \mathbb{C} \subset L^{x}$ is the complex line bundle over $T^{2}$ spanned by $\widehat{\psi^{x}} \in \hat{\mathscr{L}}_{x}$ over $x \in \Sigma$. We thus have to show that as $x$ tends to $\infty$, the line bundle $\left(1+R^{x}\right) E^{x}$ approaches the twistor lift $E \subset L$ which is the $i$-eigenspace of the complex structure $\tilde{J}$ on $L$ induced (2.1) by the conformal immersion $f$. Note that, since $\delta \in \Gamma\left(K \operatorname{End}_{+}(L, V / L)\right)$, we have $\delta E=K E_{0}$.

From $S^{\infty}=J$ we see that $S^{x}(p) \neq-J(p)$ for all $p \in T^{2}$ provided $x$ is in a sufficiently small punctured neighborhood of $\infty \in \bar{\Sigma}$. Thus, stereographic projection gives

$$
S^{x}=\left(1+Y^{x}\right) J\left(1+Y^{x}\right)^{-1}
$$

for a holomorphic family $Y^{x} \in \Gamma\left(\right.$ End $\left._{-}(V / L)\right)$ on that punctured neighborhood. The holomorphic family of flat adapted connections

$$
\nabla^{x}=\left(1+Y^{x}\right) \circ\left(\hat{\nabla}+\alpha^{x}\right) \circ\left(1+Y^{x}\right)^{-1}
$$

gauges under $1+Y^{x}$ to the holomorphic family of flat connections $\hat{\nabla}+\alpha^{x}$ so that $\alpha^{x}$ is a $\hat{\nabla}$-closed form in $\Omega^{1}\left(\operatorname{End}_{+}(V / L)\right)$. Then

$$
\eta^{x}=\frac{1}{1+\left|Y^{x}\right|^{2}}\left(\alpha^{x}+O(1)\right)
$$

and it is shown in (5.6) of [4] that

$$
\alpha^{x}=\sum_{k \geqq-1} \alpha_{k} x^{-k}
$$

has a simple pole at $\infty$ with residue $\alpha_{-1} \in H^{0}(K)$ a non-zero holomorphic 1-form on $T^{2}$. Observing that $E^{x}=\left(1+Y^{x}\right) E_{0}$, we therefore obtain from (4.1) that

$$
\left(1+R^{x}\right) E^{x}=\left(1+\delta^{-1}\left(\eta^{x}-\eta_{0}\right)\right)\left(1+Y^{x}\right) E_{0} .
$$

But this last expression tends to

$$
E=\delta^{-1} K E_{0} \subset L
$$

as $x$ tends to $\infty$, where we used that the only term which is not bounded is given by the simple pole of $\alpha^{x}$ at $\infty$.

It is precisely this theorem which will allow us to study conformal tori in the 4-sphere of finite spectral genus by algebro-geometric techniques. In [4] it is shown that the Willmore energy of $f$ can be computed in terms of residues of appropriate meromorphic forms on the compact curve $\bar{\Sigma}$. Moreover, the $T^{2}$-family of algebraic curves is in fact linear as a map into the Jacobian of $\bar{\Sigma}$. But much more is true: the Jacobian acts via flows, the DaveyStewartson hierarchy, preserving the Willmore energy of the conformal torus $f: T^{2} \rightarrow S^{4}$, and $f$ itself is the flow tangent to the Abel image of $\bar{\Sigma}$ in its Jacobian. 


\section{A. Appendix}

In this appendix we summarize the basic notions concerning the theory of quaternionic vector bundles over Riemann surfaces [11]. A quaternionic vector bundle $W$ with complex structure $J$ over a Riemann surface $M$ decomposes into

$$
W=W_{+} \oplus W_{-},
$$

where $W_{ \pm}$are the $\pm i$-eigenspaces of $J$. By restriction, $J$ induces complex structures on $W_{ \pm}$ and $W_{-}=W_{+} j$ gives a complex linear isomorphism between $W_{+}$and $W_{-}$. The degree of the quaternionic bundle $W$ with complex structure $J$ is then defined to be the degree of the underlying complex vector bundle

$$
\operatorname{deg} W:=\operatorname{deg} W_{+},
$$

which is half of the usual degree of $W$ when viewed as a complex bundle with $J$.

Given two quaternionic bundles $W$ and $\hat{W}$ with complex structures $J$ and $\hat{J}$ the complex linear homomorphisms $\operatorname{Hom}_{+}(W, \hat{W})$ are complex linearly isomorphic to

$$
\operatorname{Hom}_{\mathbb{C}}\left(W_{+}, \hat{W}_{+}\right)
$$

On the other hand, the complex antilinear homomorphisms $\operatorname{Hom}_{-}(W, \hat{W})$ are complex linearly isomorphic to $\operatorname{Hom}_{+}(\bar{W}, \hat{W})$, where the complex structure on a homomorphism bundle is induced by the target complex structure.

A quaternionic holomorphic structure on a quaternionic vector bundle $W$ with complex structure $J$ is given by a quaternionic linear operator

$$
D: \Gamma(W) \rightarrow \Gamma(\bar{K} W)
$$

satisfying the Leibniz rule $D(\psi \lambda)=(D \psi) \lambda+(\psi d \lambda)^{\prime \prime}$, where $\psi \in \Gamma(W)$ and $\lambda: M \rightarrow \mathbb{H}$. Note that $(d \lambda)^{\prime \prime}$ cannot be defined since $\mathbb{H}$ does not have a preferred complex structure, whereas $(\psi d \lambda)^{\prime \prime}$ is well defined as the $\bar{K}$-part of a $W$-valued 1 -form. The decomposition of $D$ into $J$ commuting and anticommuting parts gives

$$
D=\bar{\partial}+Q \text {. }
$$

Here $\bar{\partial}$ is the double of a complex holomorphic structure on the complex bundle $W_{+}$and $Q \in \Gamma\left(\bar{K}\right.$ End $\left._{-}(W)\right)$ is a 1 -form of type $\bar{K}$ with values in complex antilinear endomorphisms of $W$.

The quaternionic vector space of holomorphic sections of $W$ is denoted by

$$
H^{0}(W)=\operatorname{ker}(D)
$$

and has finite dimension $h^{0}(W):=\operatorname{dim} H^{0}(W)$ for compact $M$. The $L^{2}$-norm

$$
\begin{array}{r}
\mathscr{W}(W)=\mathscr{W}(W, D)=2 \underset{M}{2}\langle Q \wedge * Q\rangle \\
\text { Bereitgestellt von | Technische Universität Berlin } \\
\text { Angemeldet } \\
\text { Heruntergeladen am | 27.10.17 14:13 }
\end{array}
$$


of $Q$ is called the Willmore energy of the holomorphic bundle $W$, where $\langle\cdot, \cdot\rangle$ denotes the trace pairing on $\operatorname{End}(W)$. The special case $Q=0$, for which $\mathscr{W}(W)=0$, describes (doubles of) complex holomorphic bundles $W=W_{+} \oplus W_{+}$. A typical example of a quaternionic holomorphic structure arises from the $\bar{K}$-part $\nabla^{\prime \prime}$ of a quaternionic connection $\nabla$ on $W$.

For analytic considerations, and to make contact to the theory of Dirac operators with potentials, it is common to regard $W$ as a complex vector bundle whose complex structure $I$ is given by right multiplication by the quaternion $i$. Thus, $W=W_{+} \oplus \bar{W}_{+}$so that $Q$ is $I$-linear and $D=\bar{\partial}+Q$ has the decomposition

$$
D=\left(\begin{array}{ll}
\bar{\partial} & 0 \\
0 & \partial
\end{array}\right)+\left(\begin{array}{cc}
0 & -\bar{q} \\
q & 0
\end{array}\right)
$$

with $q \in \Gamma\left(K \operatorname{Hom}\left(W_{+}, \bar{W}_{+}\right)\right)$.

Given a linear system of a holomorphic bundle $W$, that is, a linear subspace $H \subset H^{0}(W)$, we have the Kodaira map

$$
\mathrm{ev}^{*}: W^{*} \rightarrow H^{*}
$$

Here ev denotes the bundle map which, for $p \in M$, evaluates holomorphic sections at $p$. In case the Kodaira map is injective, its image $W^{*} \subset H^{*}$ defines a map into the Grassmannian $f: M \rightarrow \operatorname{Gr}\left(r, H^{*}\right)$, where rank $W=r$. The derivative $\delta=\left.\pi d\right|_{W^{*}}$ of this map satisfies $* \delta=\delta J$, where $d$ denotes the trivial connection on $H^{*}$ and $\pi: H^{*} \rightarrow H^{*} / W^{*}$ denotes the canonical projection. In other words, $f$ is a holomorphic curve [11].

If $M$ is a compact Riemann surface of genus $g$ and $L$ is a holomorphic line bundle over $M$, the Plücker formula [11] gives a lower bound for the Willmore energy $\mathscr{W}(L)$ of $L$ in terms of the genus $g$ of $M$, the degree of $L$, and vanishing orders of holomorphic sections of $L$. For the purposes of this paper, we need a more general version of the Plücker formula which includes linear systems with monodromy. If $\tilde{M}$ is the universal cover of $M$, we denote by $\tilde{L}$ the pullback to $\tilde{M}$ of $L$ by the covering map. The holomorphic structure of $L$ lifts to make $\tilde{L}$ into a holomorphic line bundle.

A linear system with monodromy is a linear subspace $H \subset H^{0}(\tilde{L})$ of holomorphic sections of $\tilde{L}$ such that

$$
\gamma^{*} H=H \quad \text { for all } \gamma \in \pi_{1}(M)
$$

where $\pi_{1}(M)$ acts via deck transformations. Adapting the proof in [11] by replacing the trivial connection with a flat connection on $H^{*}$, we obtain the Plücker formula for an $n$-dimensional linear system $H$ with monodromy

$$
\frac{1}{4 \pi} \mathscr{W}(L) \geqq n((n-1)(1-g)-\operatorname{deg} L)+\operatorname{ord} H .
$$

The order ord $H$ of the linear system $H \subset H^{0}(\tilde{L})$ with monodromy is computed as follows: to a point $x \in \tilde{M}$ we assign the Weierstrass gap sequence $n_{0}(x)<\cdots<n_{n-1}(x)$ inductively 
by letting $n_{k}(x)$ be the minimal vanishing order strictly greater than $n_{k-1}(x)$ of holomorphic sections in $H$. Away from isolated points this sequence is $n_{k}(x)=k$ and

$$
\operatorname{ord}_{x} H=\sum_{k=0}^{n-1}\left(n_{k}-k\right)
$$

measures the deviation from the generic sequence. Since $H$ is a linear system with monodromy, the Weierstrass gap sequence is invariant under deck transformations. Therefore, $\operatorname{ord}_{p} H$ for $p \in M$ is well defined, zero away from finitely many points, and the order of the linear system $H$ is given by

(A.11) $\quad$ ord $H=\sum_{p \in M} \operatorname{ord}_{p} H$.

For a linear system $H \subset H^{0}(L)$ without monodromy, formula (A.10) is the usual Plücker relation as discussed in [11].

\section{References}

[1] L. Bianchi, Il teorema di permutabilità per le trasformazioni di Darboux delle superficie isoterme, Rom. Acc. L. Rend. 13 (1904), 359-367.

[2] A. Bobenko, All constant mean curvature tori in $R^{3}, S^{3}, H^{3}$ in terms of theta functions, Math. Ann. 290 (1991), 209-245.

[3] C. Bohle, Constrained Willmore tori in the 4-sphere, J. Diff. Geom., to appear.

[4] C. Bohle, F. Pedit and U. Pinkall, Spectral curves of quaternionic holomorphic line bundles over 2-tori, Manuscri. Math. 130 (2009), 311-352.

[5] J. Bolton, F. Pedit and L. M. Woodward, Minimal surfaces and the affine Toda field model, J. reine angew. Math. 459 (1995), 119-150.

[6] F. Burstall, D. Ferus, K. Leschke, F. Pedit and U. Pinkall, Conformal geometry of surfaces in $S^{4}$ and quaternions, Lect. Notes Math. 1772, Springer-Verlag, Berlin 2002.

[7] F. Burstall, D. Ferus, F. Pedit and U. Pinkall, Harmonic tori in symmetric spaces and commuting Hamiltonian systems on loop algebras, Ann. Math. (2) 138 (1993), 173-212.

[8] G. Darboux, Sur les surfaces isothermiques, C. R. Acad. Sci. Paris 128 (1899), 1299-1305.

[9] A. Davey and K. Stewartson, On three-dimensional packets of surface waves, Proc. R. Soc. Lond. Ser. A Math. Phys. Eng. 338 (1974), 101-110.

[10] J. Feldmann, H. Knörrer and E. Trubowitz, Infinite genus Riemann surfaces, American Mathematical Society, Providence, RI, 2003.

[11] D. Ferus, K. Leschke, F. Pedit and U. Pinkall, Quaternionic holomorphic geometry: Plücker formula, Dirac eigenvalue estimates and energy estimates of harmonic 2-tori, Invent. Math. 146 (2001), 507-559.

[12] D. Ferus, F. Pedit, U. Pinkall and I. Sterling, Minimal tori in $S^{4}$, J. reine angew. Math. 429 (1992), 1-47.

[13] P. G. Grinevich and M. U. Schmidt, Conformal invariant functionals of immersions of tori into $R^{3}$, J. Geom. Phys. 26 (1998), 51-78.

[14] U. Hertrich-Jeromin, Introduction to Möbius differential geometry, Cambridge University Press, Cambridge 2003.

[15] N. Hitchin, Harmonic maps from a 2-torus to the 3-sphere, J. Diff. Geom. 31 (1990), 627-710.

[16] M. Kilian and M. Schmidt, On the moduli of constant mean curvature cylinders of finite type in the 3-sphere, preprint 2007, http://www.arxiv.org/abs/0712.0108.

[17] I. Krichever, Spectral theory of two-dimensional periodic operators and its applications, Russian Math. Surveys 44 (1989), 145-225.

[18] K. Leschke, F. Pedit and U. Pinkall, Willmore tori in the 4-sphere with nontrivial normal bundle, Math. Ann. 332 (2005), 381-394.

[19] V. Marchenko, Sturm-Liouville operators and their applications (Russian), Izdat. "Naukova Dumka", Kiev 1977. 
[20] F. Pedit and U. Pinkall, Quaternionic analysis on Riemann surfaces and differential geometry, Proceedings of the International Congress of Mathematicians, Volume II (Berlin 1998), Documenta mathematica, Extra Volume ICM (1998), 389-400.

[21] U. Pinkall and I. Sterling, On the classification of constant mean curvature tori, Ann. Math. (2) 130 (1989), 407-451.

[22] M. U. Schmidt, A proof of the Willmore conjecture, preprint 2002, http://www.arxiv.org/abs/math/0203224.

[23] I. A. Taimanov, The Weierstrass representation of closed surfaces in $\mathbb{R}^{3}$, Funct. Anal. Appl. 32 (1998), 49-62.

Institut für Mathematik, Technische Universität Berlin, Straße des 17. Juni 136, 10623 Berlin, Germany e-mail: bohle@math.tu-berlin.de

Department of Mathematics, University of Leicester, Leicester, LE1 7RH, UK

e-mail: k.leschke@mcs.le.ac.uk

Mathematisches Institut der Universität Tübingen, Auf der Morgenstunde 10, 72076 Tübingen, Germany and

Department of Mathematics, University of Massachusetts, Amherst, MA 01003, USA

e-mail: pedit@mathematik.uni-tuebingen.de

Institut für Mathematik, Technische Universität Berlin, Straße des 17. Juni 136, 10623 Berlin, Germany e-mail: pinkall@math.tu-berlin.de

Eingegangen 7. Januar 2009 